\title{
Anion-controlled positional switching of a phenyl group about the dinuclear core of a AuSb complex
}

\author{
Srobona Sen, Iou Sheng Ke and François P. Gabbai* \\ Department of Chemistry, Texas A\&M University, College Station, TX 77843-3255.
}

\section{SUPPORTING INFORMATION}

This PDF file includes:

Figure S1. ${ }^{1} \mathrm{H}$ NMR spectrum of 2 in $\mathrm{CDCl}_{3}$

Figure S2. ${ }^{13} \mathrm{C}$ NMR spectrum of $\mathbf{2}$ in $\mathrm{CDCl}_{3}$

Figure S3. ${ }^{31} \mathrm{P}$ NMR spectrum of $\mathbf{2}$ in $\mathrm{CDCl}_{3}$

Figure S4. ${ }^{1} \mathrm{H}$ NMR spectrum of 3 in $\mathrm{CDCl}_{3}$

Figure S5. ${ }^{13} \mathrm{C}$ spectrum of 3 in $\mathrm{CDCl}_{3}$

Figure S6. ${ }^{31} \mathrm{P}$ NMR spectrum of $\mathbf{3}$ in $\mathrm{CDCl}_{3}$

Figure S7. ${ }^{19} \mathrm{~F}$ NMR spectrum of 3 in $\mathrm{CDCl}_{3}$

Figure S8. ${ }^{31} \mathrm{P}$ NMR spectrum of 4 in $\mathrm{CDCl}_{3}$

Figure S9. ${ }^{19} \mathrm{~F}$ NMR spectrum of $\mathbf{4}$ in $\mathrm{CDCl}_{3}$

Figure S10. ${ }^{1} \mathrm{H}$ spectrum of 5 in $\mathrm{CDCl}_{3}$

Figure S11. ${ }^{13} \mathrm{C}$ spectrum of 5 in $\mathrm{CDCl}_{3}$

Figure S12. ${ }^{31} \mathrm{P}$ spectrum of 5 in $\mathrm{CDCl}_{3}$

Figure S13. ${ }^{19} \mathrm{~F}$ spectrum of 5 in $\mathrm{CDCl}_{3}$

Figure S14. ${ }^{31} \mathrm{P}$ spectrum of $\mathbf{6}$ in $\mathrm{CDCl}_{3}$

Figure S15. ${ }^{19} \mathrm{~F}$ spectrum of $\mathbf{6}$ in $\mathrm{CDCl}_{3}$

Figure S16. ${ }^{1} \mathrm{H}$ NMR spectrum of 7 in $\mathrm{CDCl}_{3}$

Figure S17. ${ }^{13} \mathrm{C}$ spectrum of 7 in $\mathrm{CDCl}_{3}$

Figure S18. ${ }^{31} \mathrm{P}$ NMR spectrum of 7 in $\mathrm{CDCl}_{3}$

Figure S19. ${ }^{19} \mathrm{~F}$ NMR spectrum of 7 in $\mathrm{CDCl}_{3}$

Figure S20. Conversion of $\mathbf{3}$ to $\mathbf{2}$ observed by ${ }^{31} \mathrm{P}$ NMR

Figure S21. Conversion of $\mathbf{3}$ to $\mathbf{2}$ observed by ${ }^{19} \mathrm{~F}$ NMR

Figure S22. Bond paths and BCPs found for 4 using QTAIM and LOL map of 4 in the plane of Sb-Au-P 
Figure S23. Histogram of the Au- $\mathrm{C}_{\mathrm{Ph}}$ bond distances found in the Cambridge Structural Database using Mogul 1.7.1

Table S1-6: XYZ Coordinates of the optimized structures of the complexes 2, 3, 4, 5, 6 and 7

Table S7. Selected bond lengths $(\AA)$ and angles $\left(^{\circ}\right)$ for complexes $\mathbf{2 , 3 , 4 , 5}$ and 7 as determined crystallographically and optimized computationally.

Table S8. Selected bond lengths $(\AA)$ and angles $\left(^{\circ}\right)$ for complex 6 as determined crystallographically and optimized computationally. 


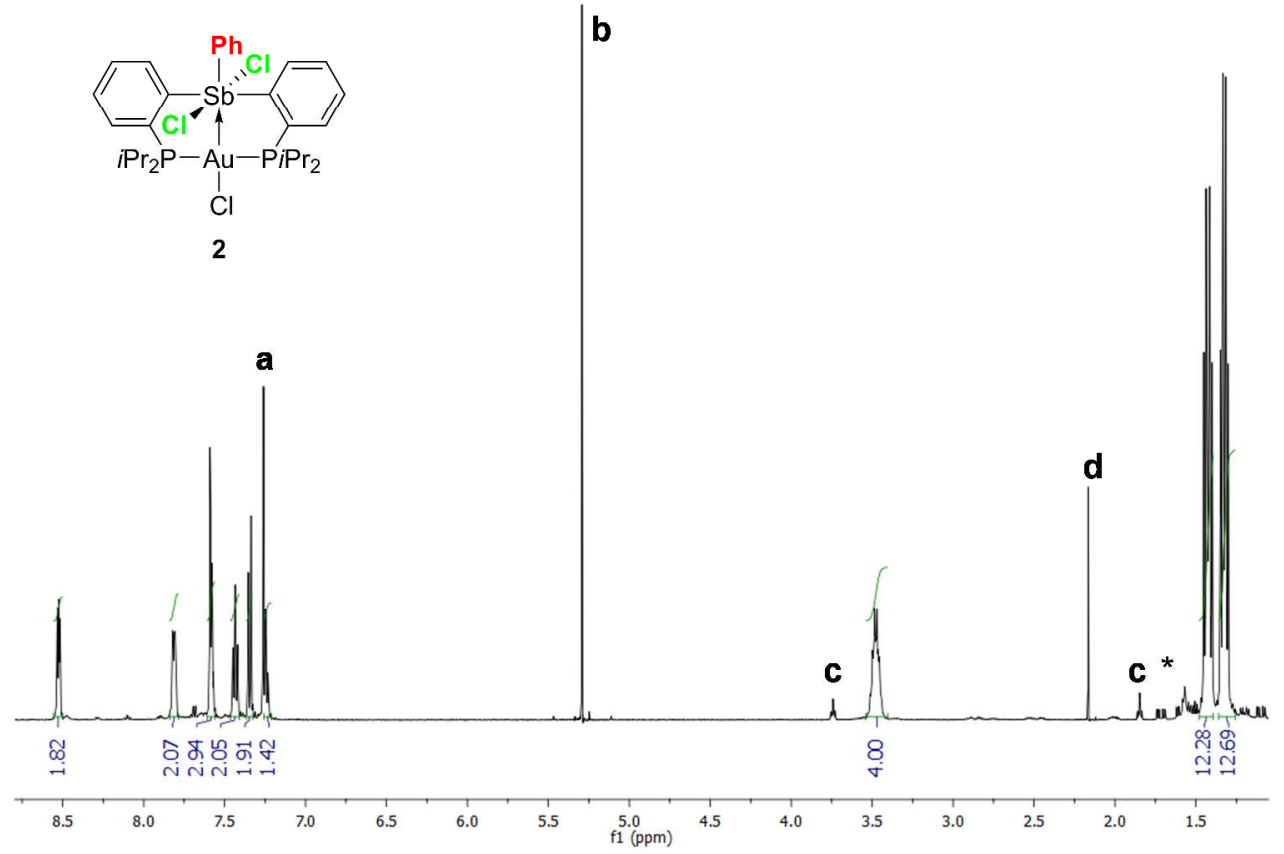

Figure S1. ${ }^{1} \mathrm{H}$ NMR spectrum of 2 in $\mathrm{CDCl}_{3}$. Residual solvent peaks are shown in the spectrum. a) $\mathrm{CHCl}_{3}, \mathrm{~b}$ ) $\left.\left.\mathrm{CH}_{2} \mathrm{Cl}_{2}, \mathrm{c}\right) \mathrm{THF}, \mathrm{d}\right)$ Acetone, $*$ denotes impurities

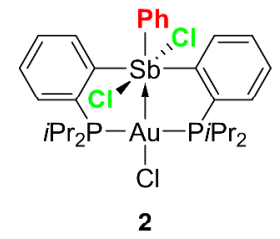

a

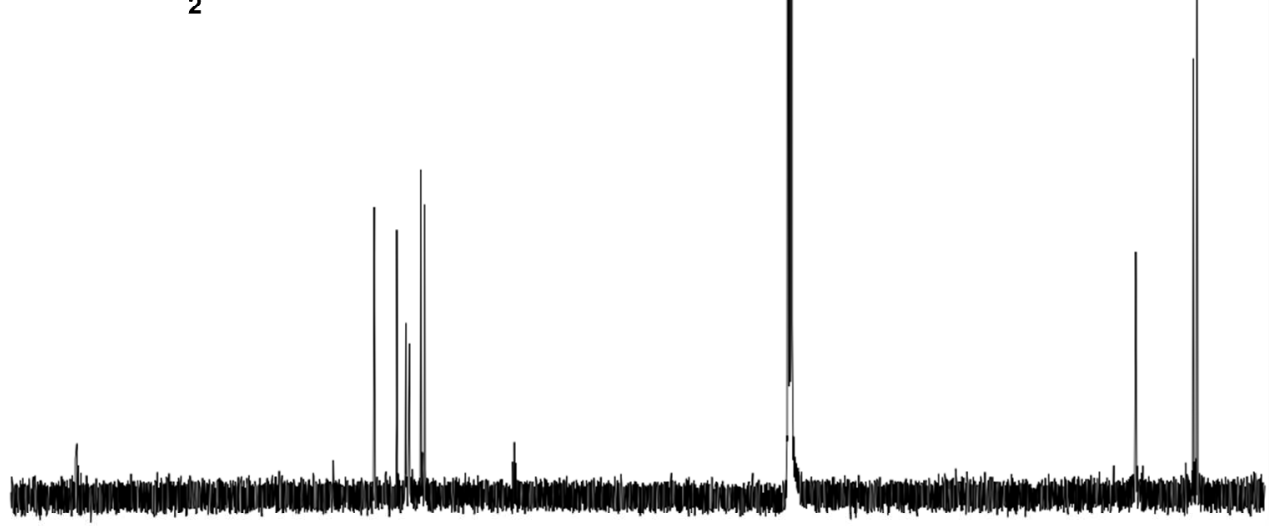

$\begin{array}{llllllllllllllllll}180 & 170 & 160 & 150 & 140 & 130 & 120 & 110 & 100 & 1 & 8 & 1 & 1 & 1 & 1 & 1 \\ \mathrm{f} 1(\mathrm{ppm}) & 90 & 80 & 70 & 60 & 50 & 40 & 30 & 20 & 10\end{array}$

Figure S2. ${ }^{13} \mathrm{C}$ NMR spectrum of $\mathbf{2}$ in $\mathrm{CDCl}_{3}$. Residual solvent peak is shown in the spectrum. a) $\mathrm{CDCl}_{3}$ 

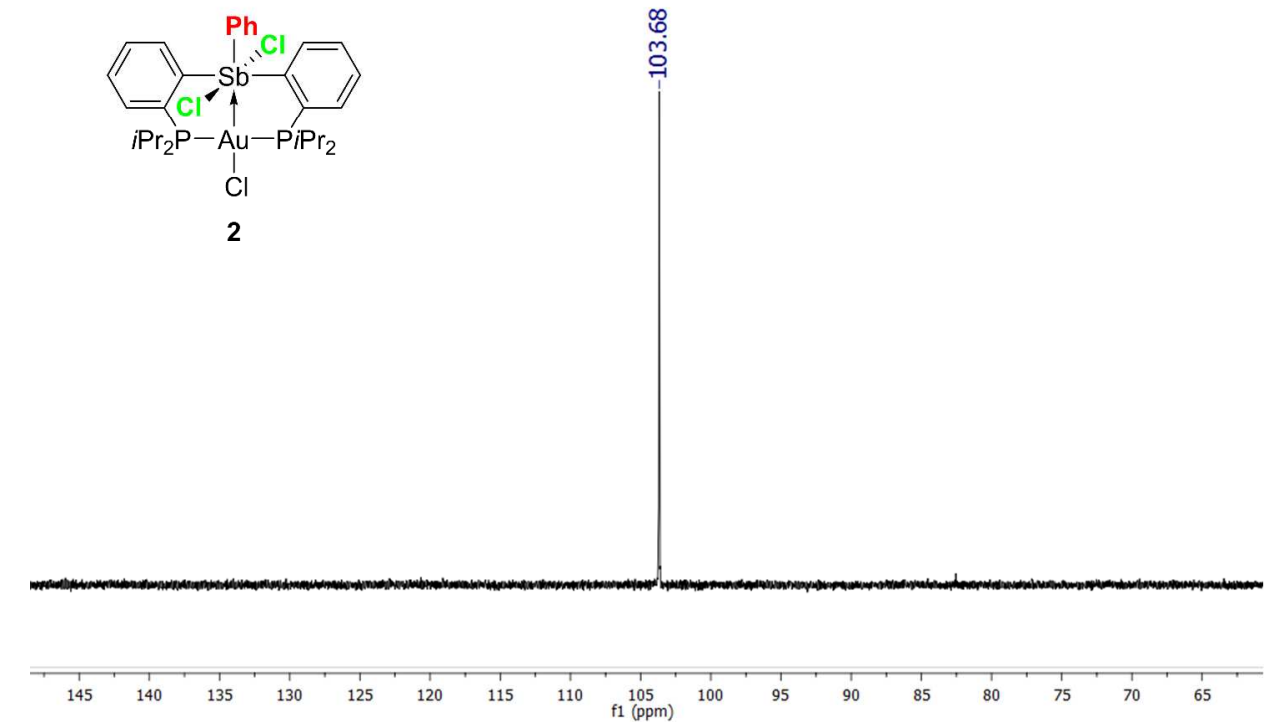

Figure S3. ${ }^{31} \mathrm{P}$ NMR spectrum of 2 in $\mathrm{CDCl}_{3}$

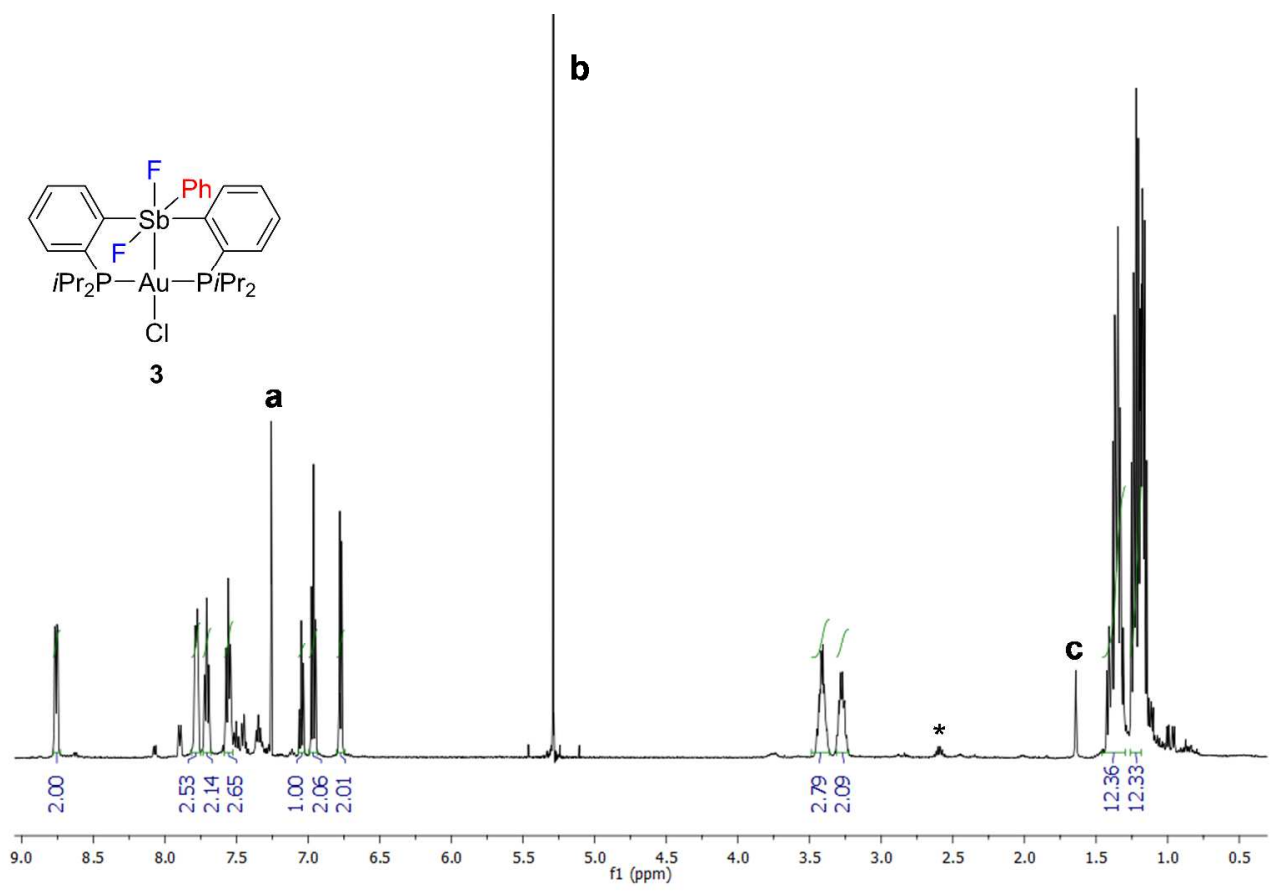

Figure S4. ${ }^{1} \mathrm{H}$ NMR spectrum of 3 in $\mathrm{CDCl}_{3}$. Residual solvent peaks are shown in the spectrum. a) $\mathrm{CHCl}_{3}, \mathrm{~b}$ ) $\left.\mathrm{CH}_{2} \mathrm{Cl}_{2}, \mathrm{c}\right) \mathrm{H}_{2} \mathrm{O}$. * denotes impurities 


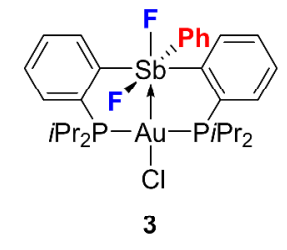

a

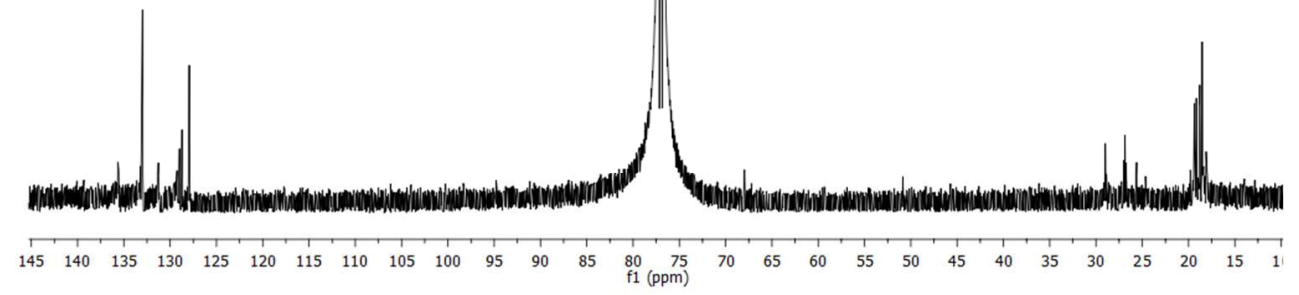

Figure S5. ${ }^{13} \mathrm{C}$ NMR spectrum of $\mathbf{3}$ in $\mathrm{CDCl}_{3}$. Residual solvent peak is shown in the spectrum. a) $\mathrm{CDCl}_{3}$
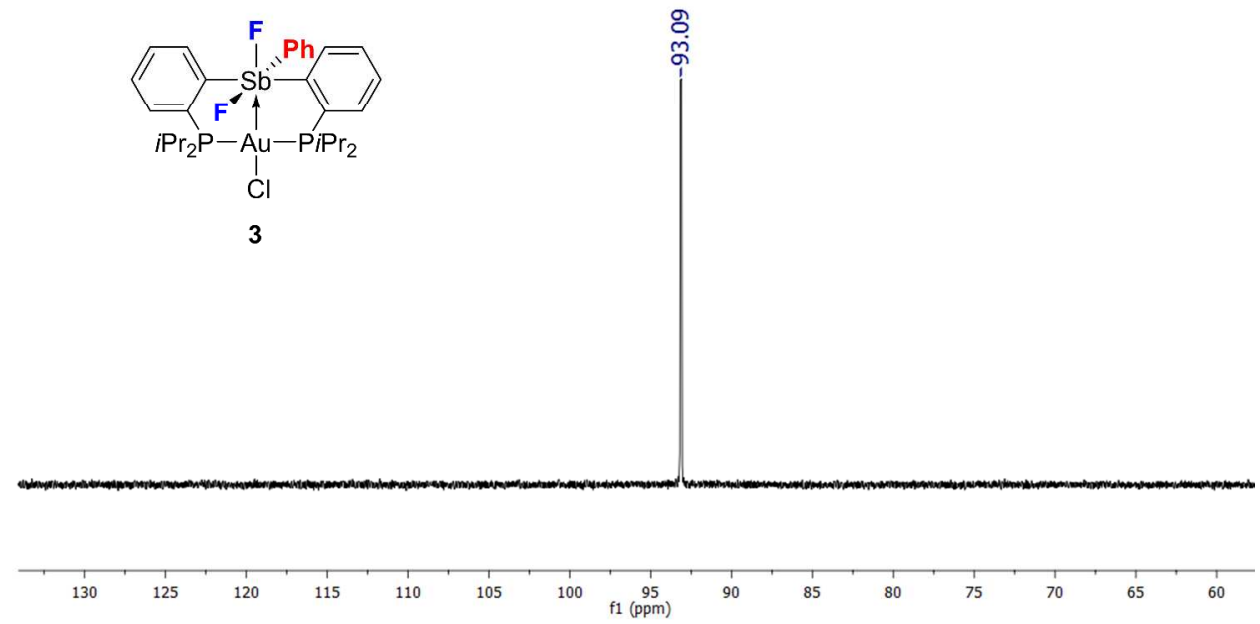

Figure S6. ${ }^{31} \mathrm{P}$ NMR spectrum of $\mathbf{3}$ in $\mathrm{CDCl}_{3}$ 


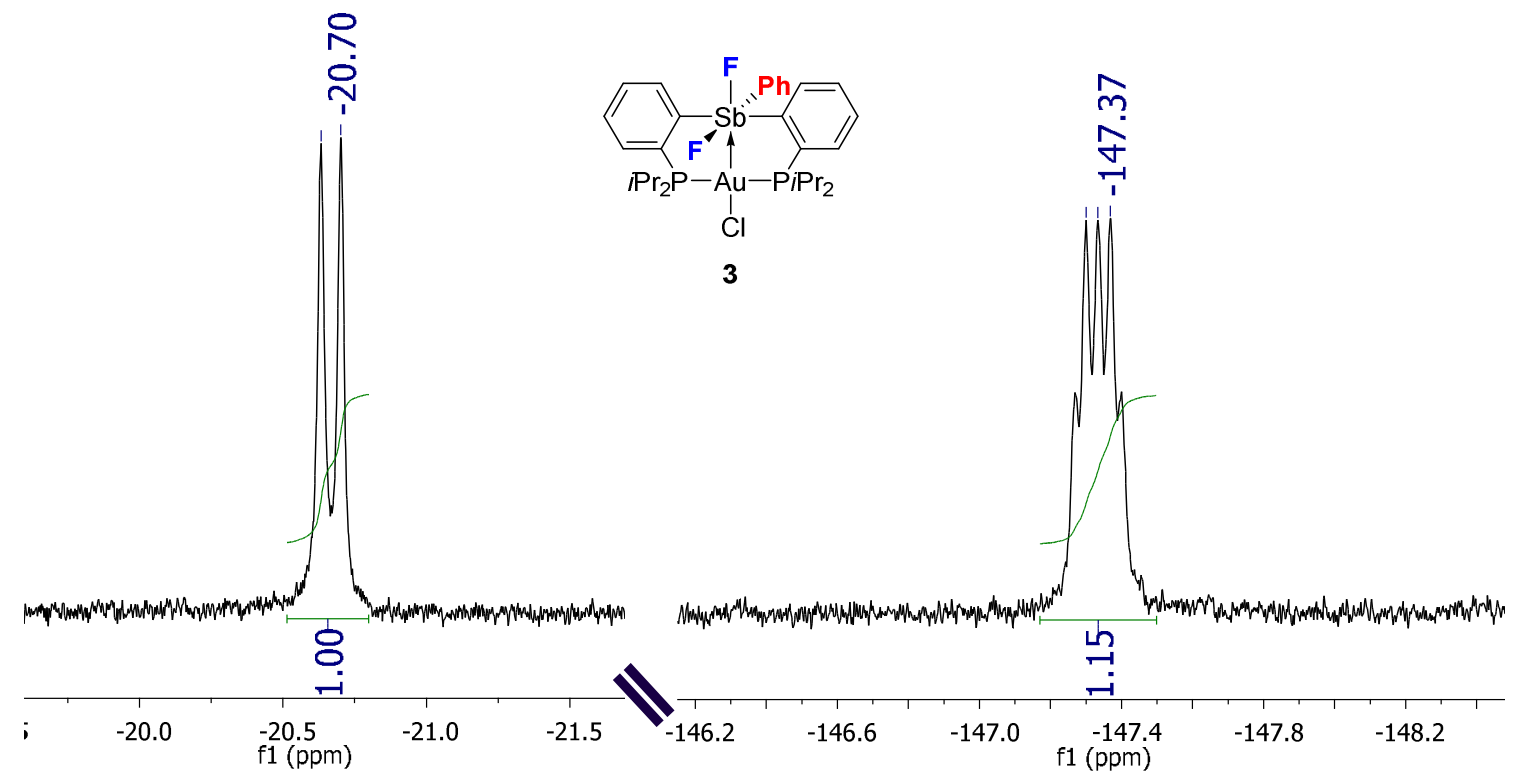

Figure S7. ${ }^{19} \mathrm{~F}$ NMR spectrum of 3 in $\mathrm{CDCl}_{3}$

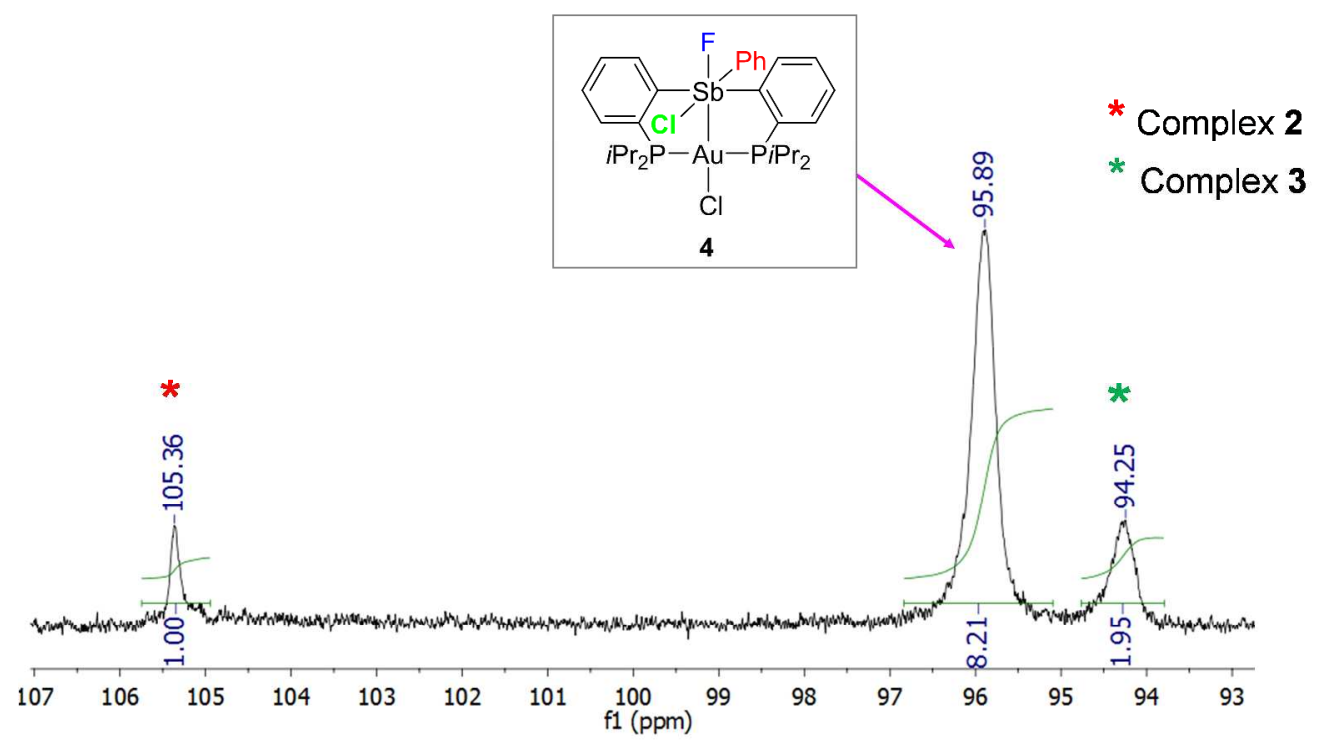

Figure S8. ${ }^{31} \mathrm{P}$ NMR of an equimolar mixture of complex 3 and $t$-Butyldimethylsilyl fluoride in $\mathrm{CH}_{2} \mathrm{Cl}_{2}$ after $24 \mathrm{hr}$. 


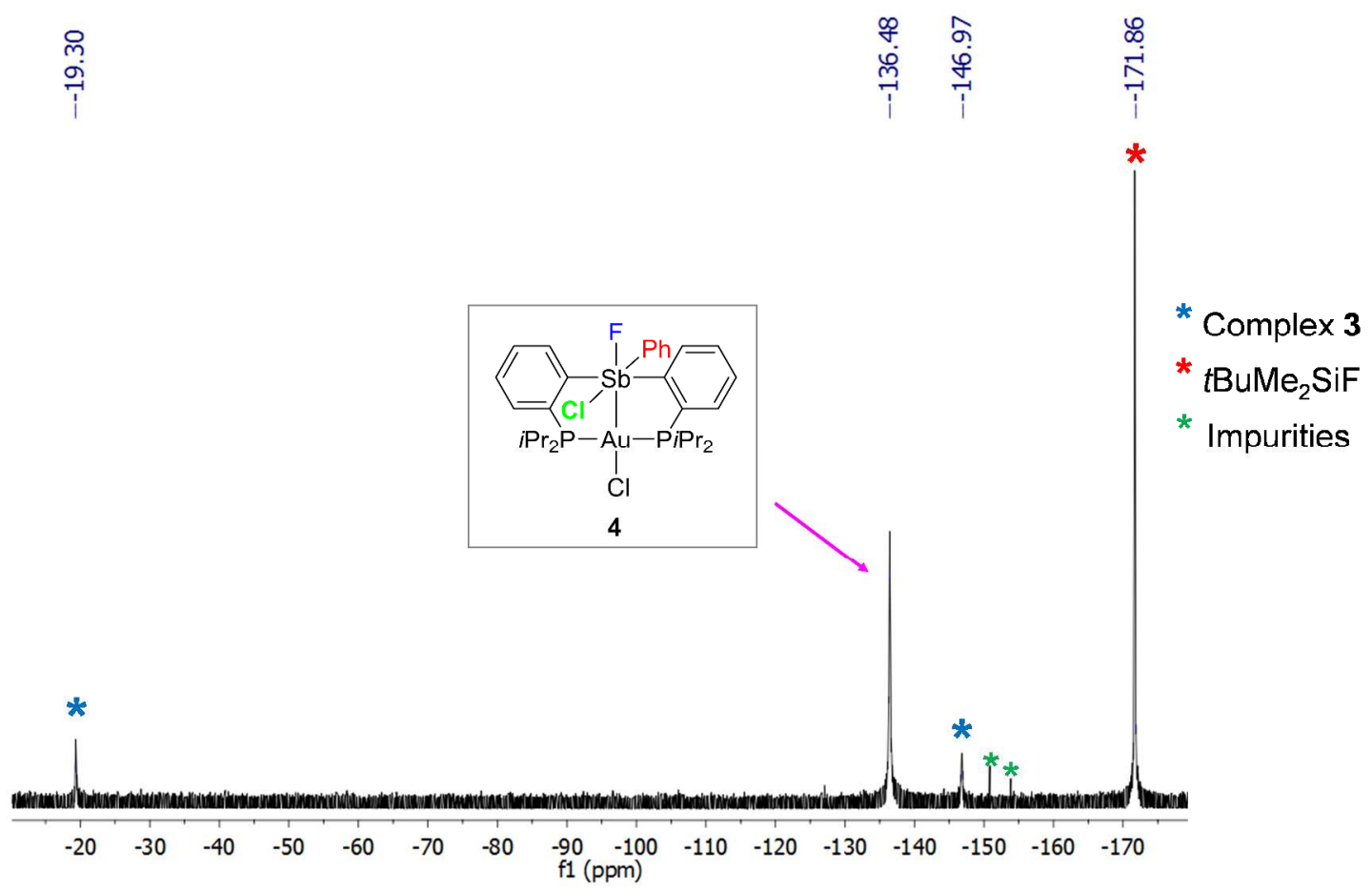

Figure S9. ${ }^{19} \mathrm{~F}$ NMR of an equimolar mixture of complex 3 and $t$-Butyldimethylsilyl fluoride in $\mathrm{CH}_{2} \mathrm{Cl}_{2}$ after $24 \mathrm{hr}$.
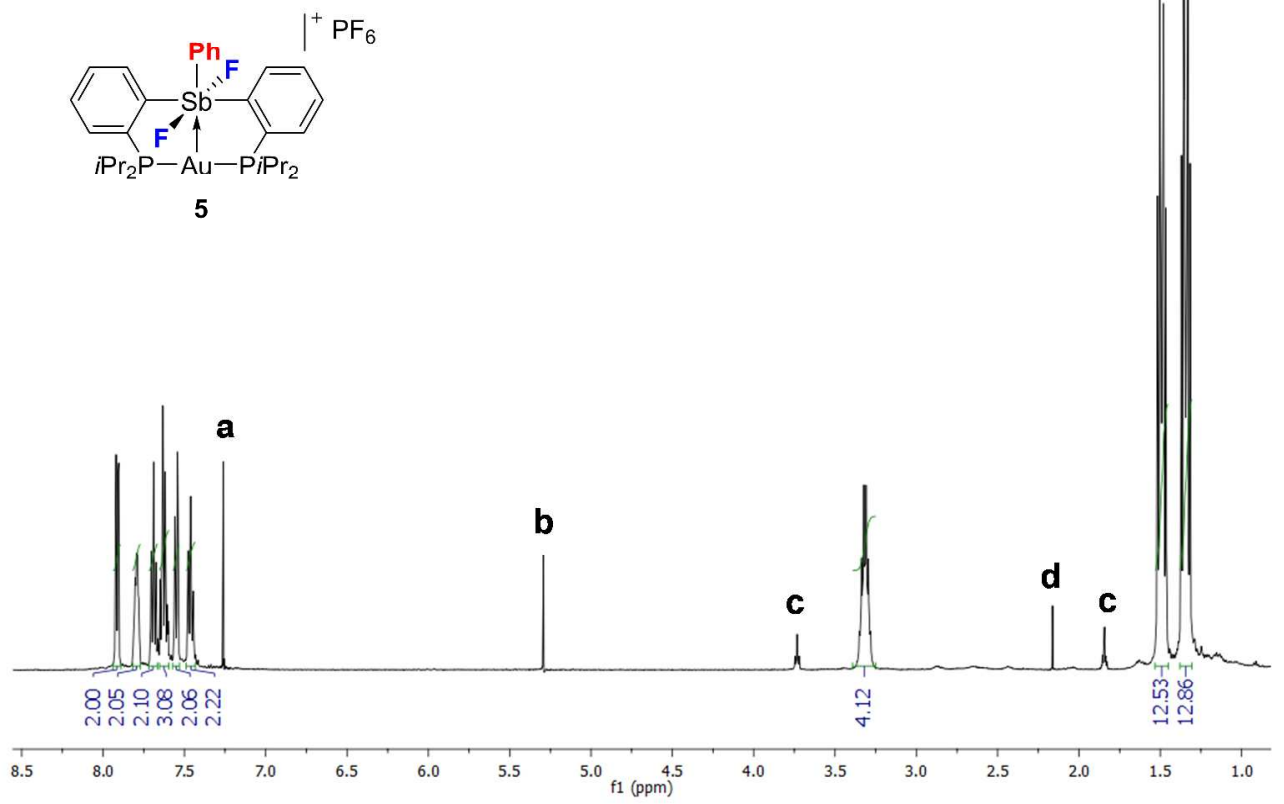

Figure S10. ${ }^{1} \mathrm{H}$ NMR spectrum of 5 in $\mathrm{CDCl}_{3}$. Residual solvent peaks are shown in the spectrum. a) $\mathrm{CHCl}_{3}, \mathrm{~b}$ ) $\left.\left.\mathrm{CH}_{2} \mathrm{Cl}_{2}, \mathrm{c}\right) \mathrm{THF}, \mathrm{d}\right)$ Acetone 

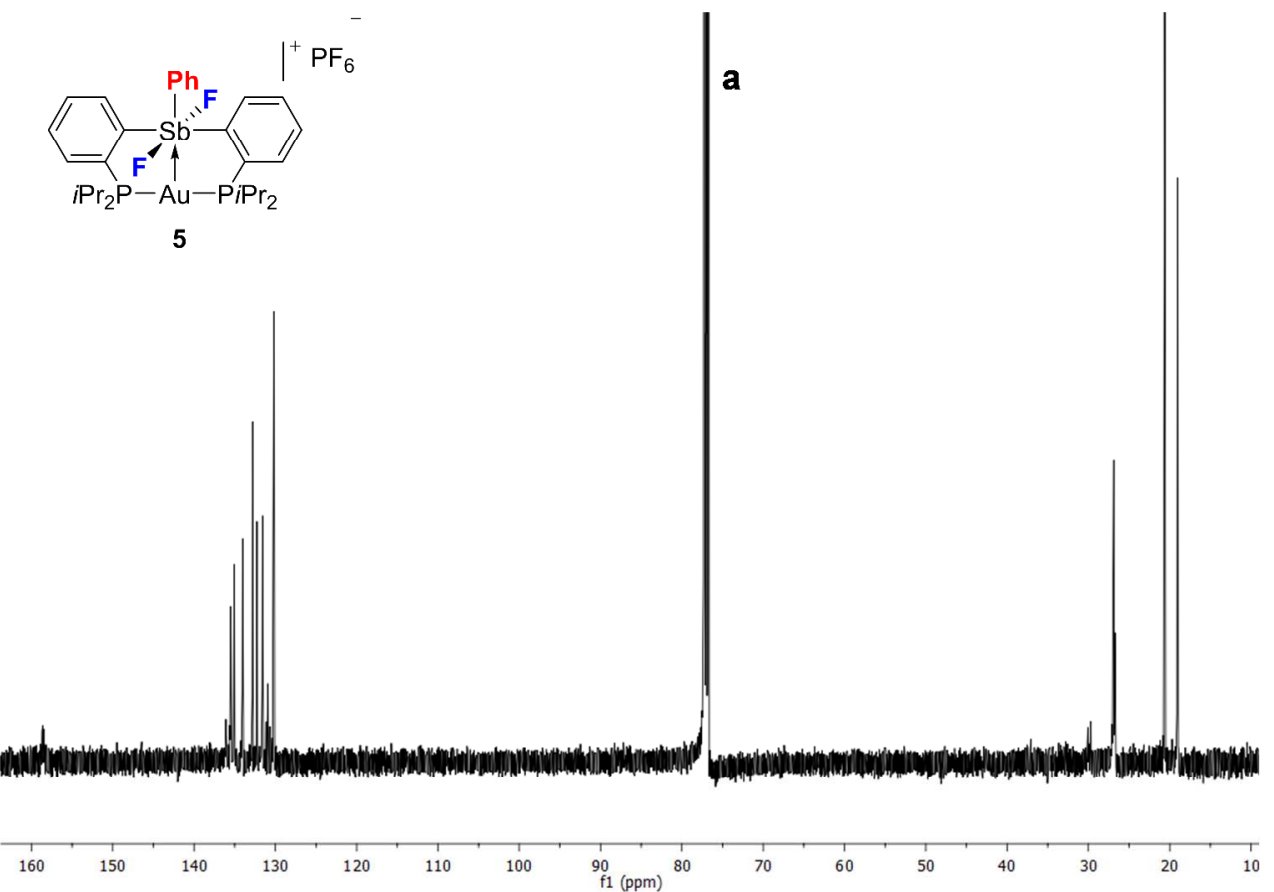

Figure S11. ${ }^{13} \mathrm{C}$ NMR spectrum of 5 in $\mathrm{CDCl}_{3}$. Residual solvent peak is shown in the spectrum. a) $\mathrm{CDCl}_{3}$
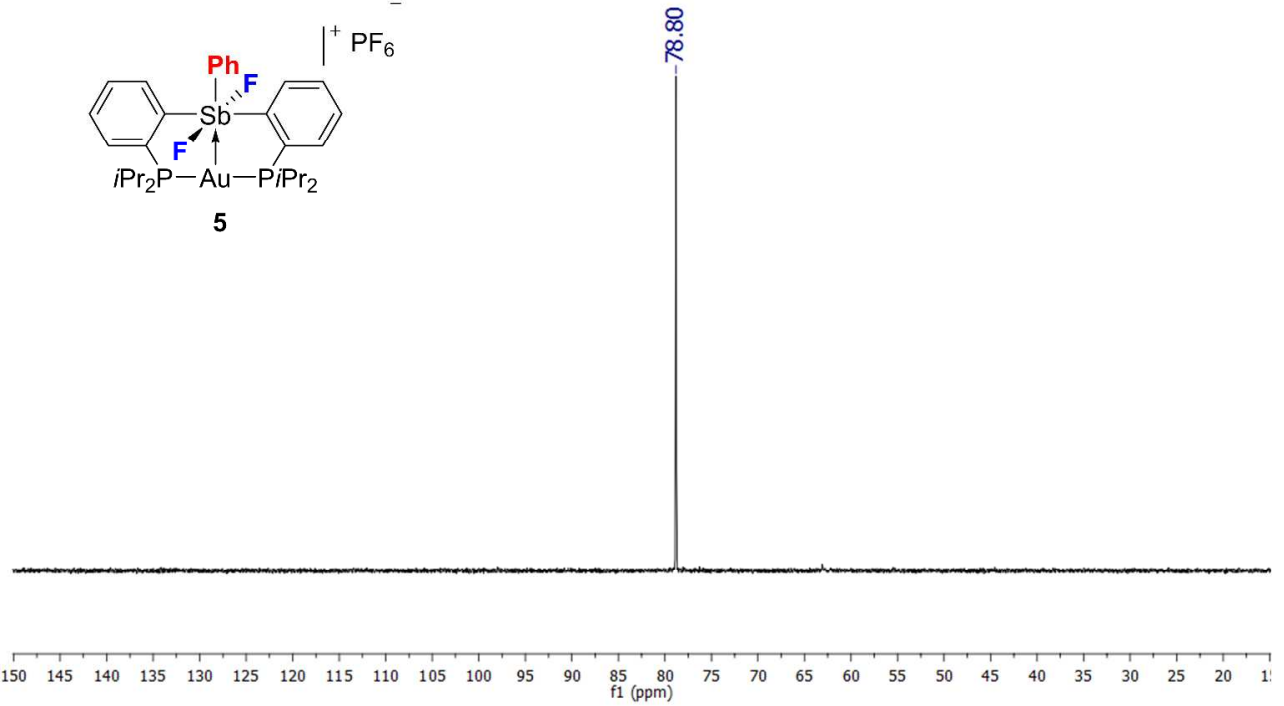

Figure S12. ${ }^{31} \mathrm{P}$ NMR spectrum of 5 in $\mathrm{CDCl}_{3}$ 


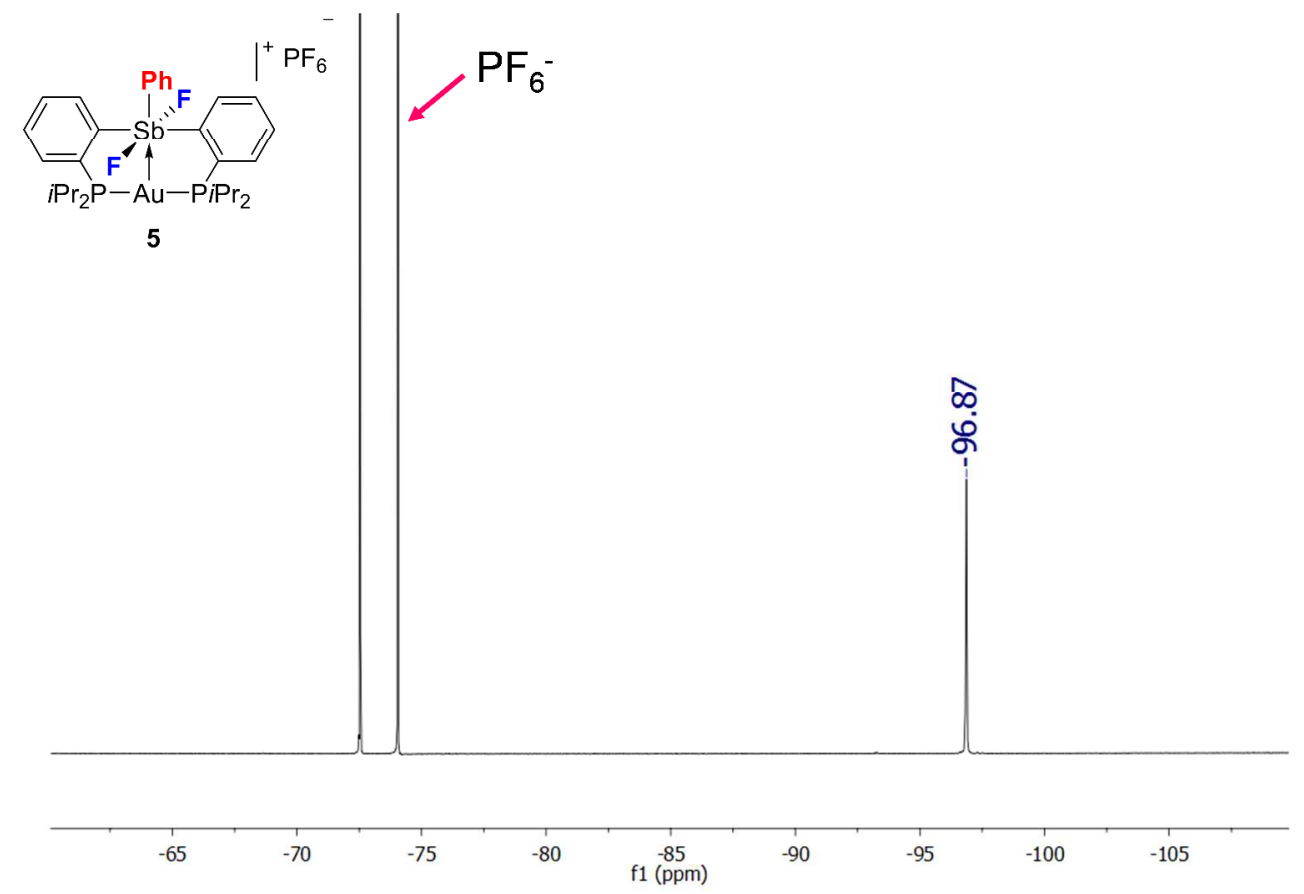

Figure S13. ${ }^{19} \mathrm{~F}$ NMR spectrum of 5 in $\mathrm{CDCl}_{3}$
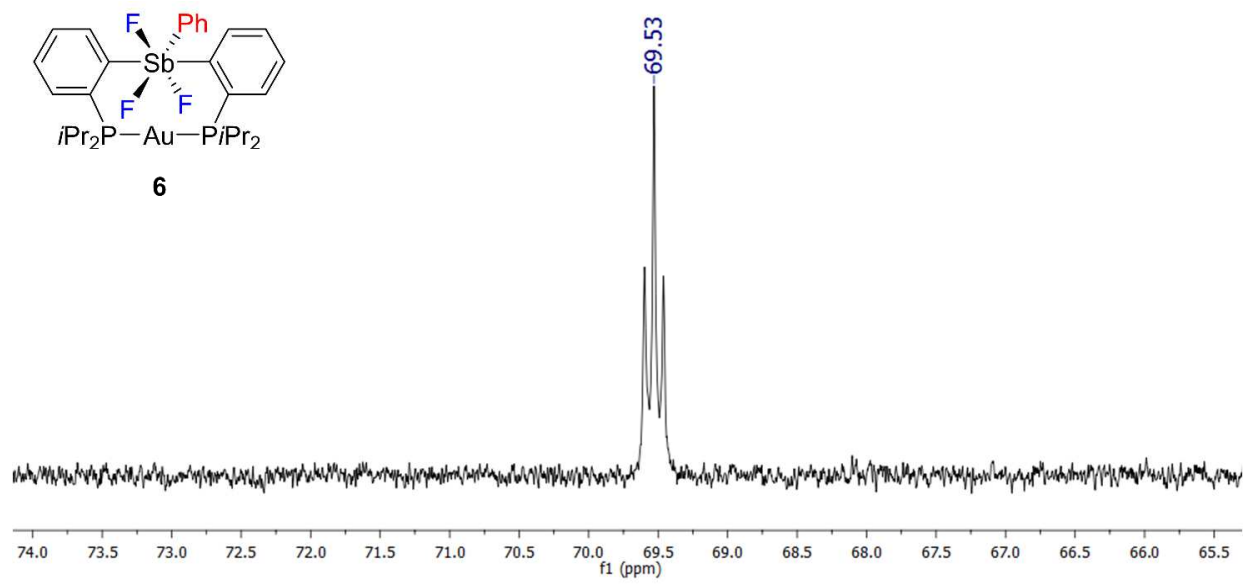

Figure S14. ${ }^{31} \mathrm{P}$ NMR spectrum of 6 in $\mathrm{CDCl}_{3}$ 


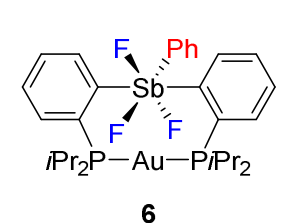

6

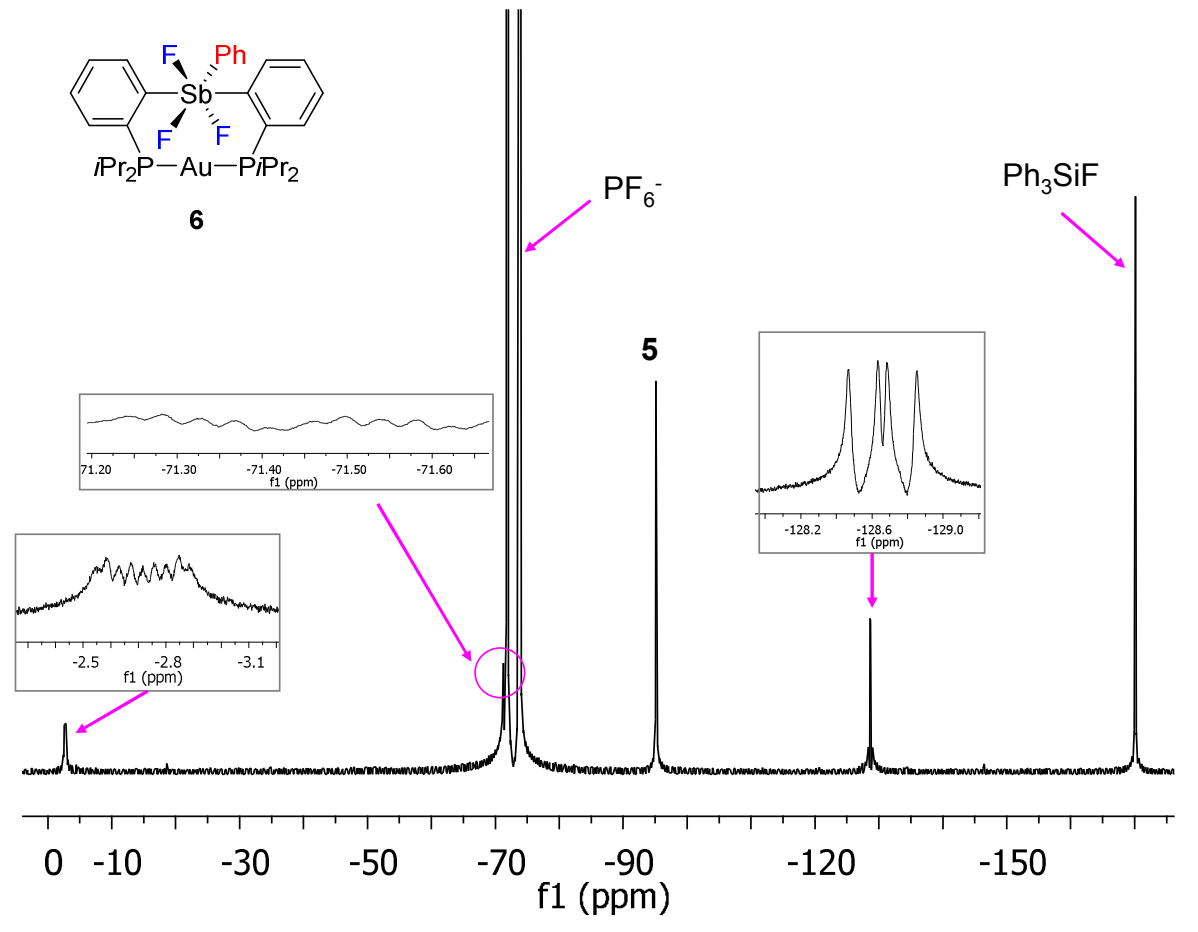

Figure S15. ${ }^{19} \mathrm{~F}$ NMR spectrum of 6 in $\mathrm{CDCl}_{3}$. The insets correspond to the antimony-bound fluorine atoms. These insets correspond to the signals shown in the Figure 11 of the main text. However, in figure 11, the phase of each resonance was individually optimized within the selected spectral window.

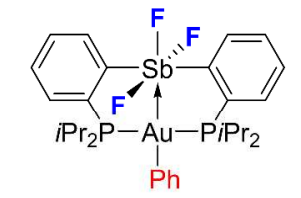

7

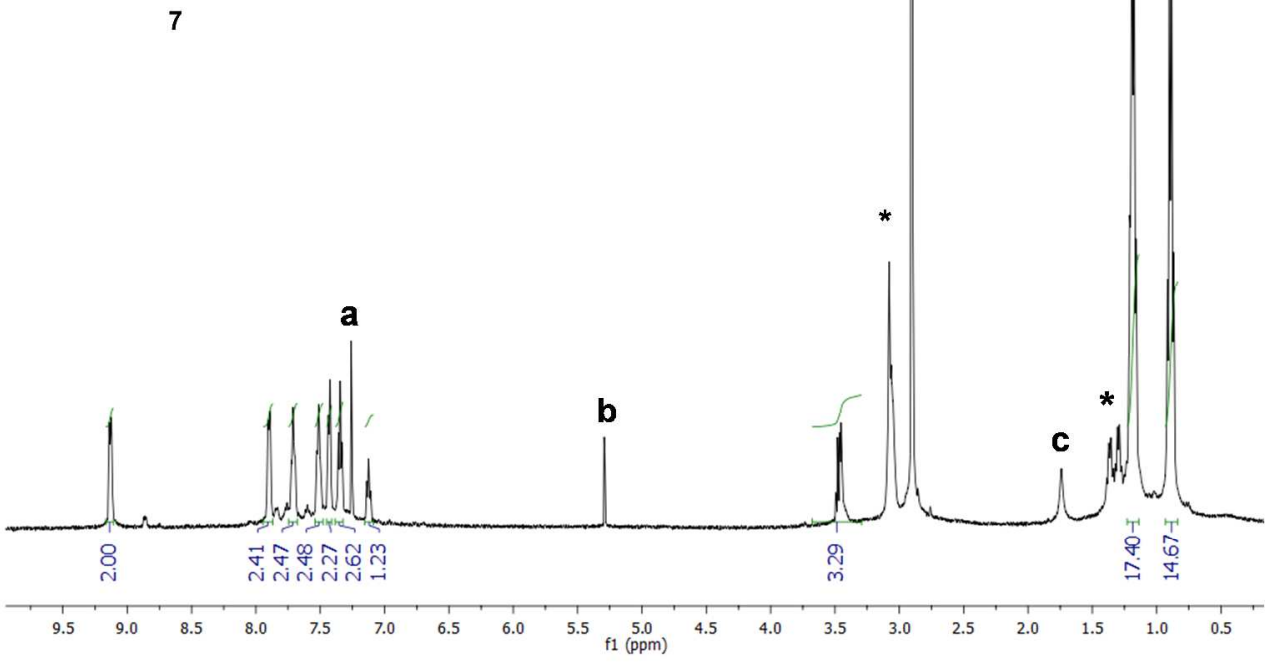

Figure S16. ${ }^{1} \mathrm{H}$ NMR spectrum of 7 in $\mathrm{CDCl}_{3}$. Residual solvent peaks are shown in the spectrum. a) $\mathrm{CHCl}_{3}$, b) $\left.\mathrm{CH}_{2} \mathrm{Cl}_{2}, \mathrm{c}\right) \mathrm{H}_{2} \mathrm{O}$. * denotes unidentified impurities 


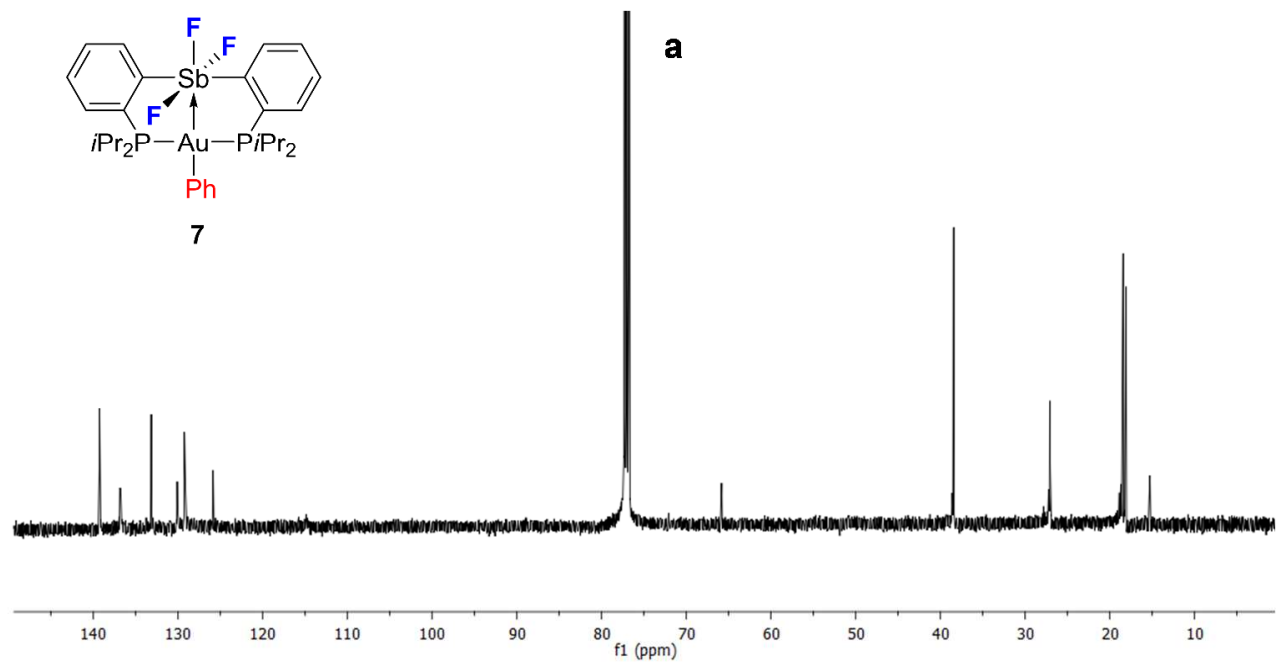

Figure S17. ${ }^{13} \mathrm{C}$ NMR spectrum of 7 in $\mathrm{CDCl}_{3}$. Residual solvent peak is denoted in the spectrum. a) $\mathrm{CDCl}_{3}$

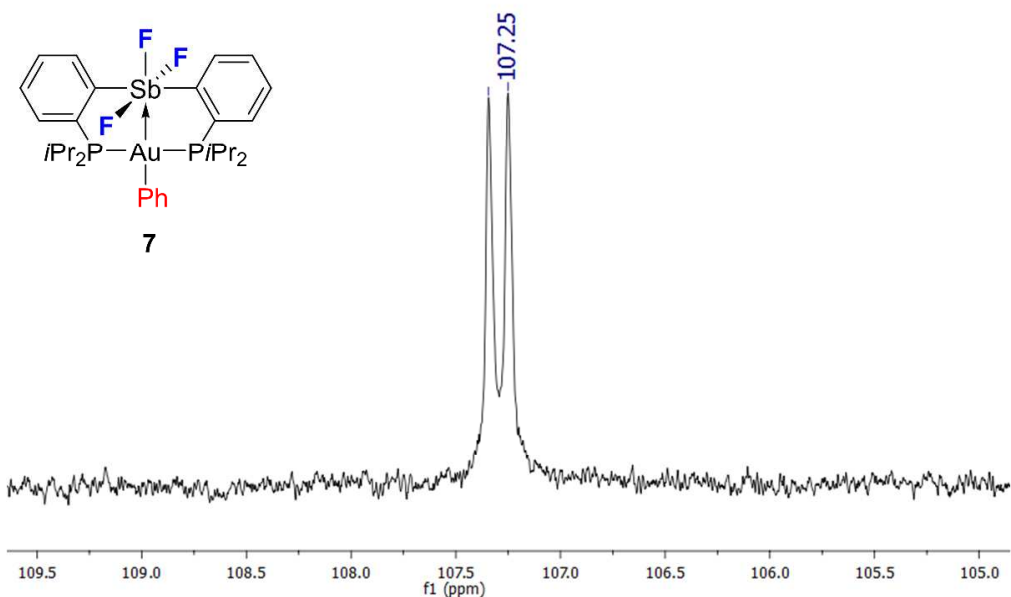

Figure S18. ${ }^{31} \mathrm{P}$ NMR spectrum of 7 in $\mathrm{CDCl}_{3}$ 


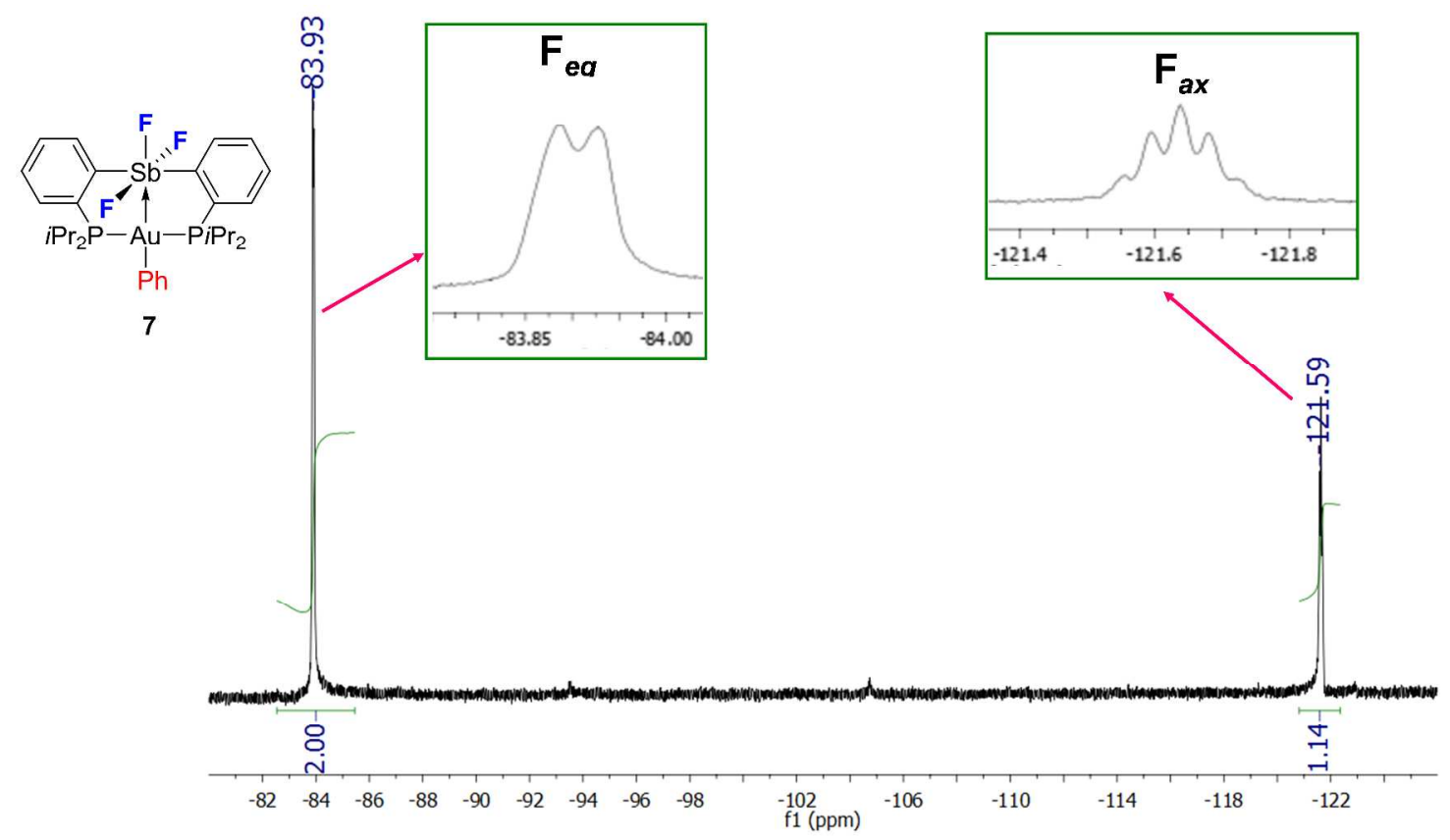

Figure S19. ${ }^{19} \mathrm{~F}$ NMR spectrum of 7 in $\mathrm{CDCl}_{3}$
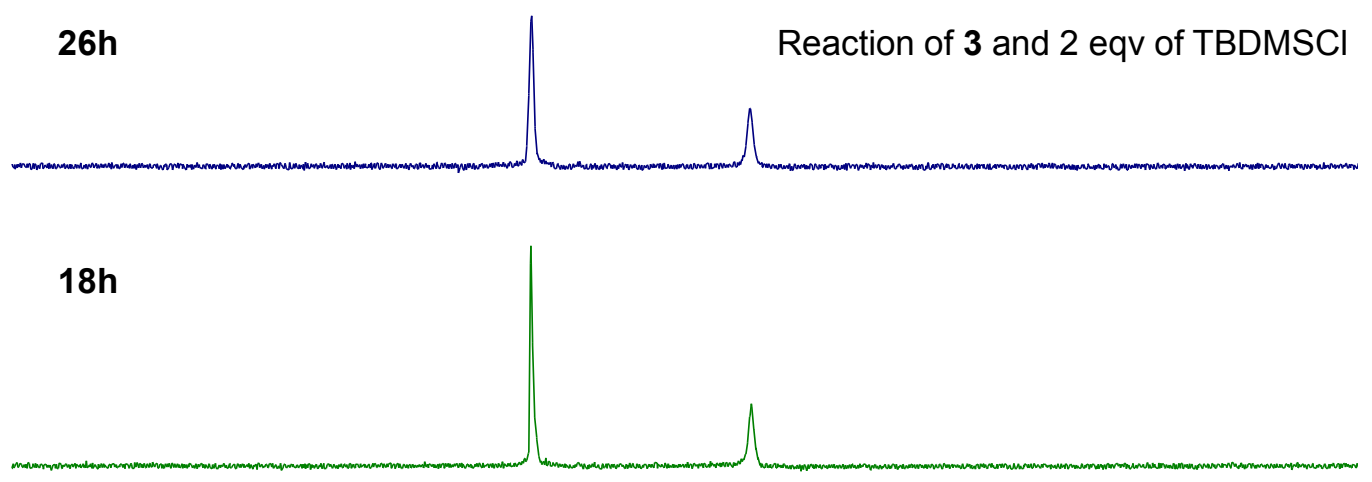

\section{$0.5 \mathrm{~h}$}

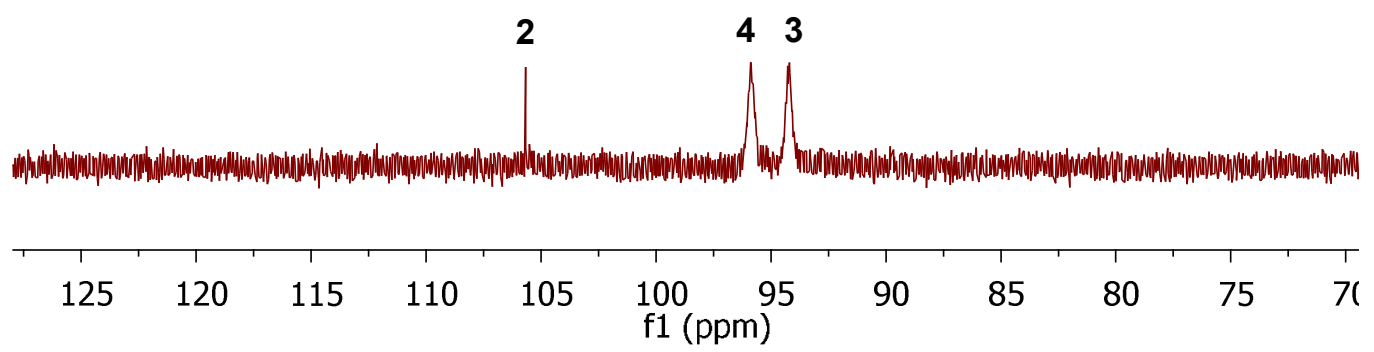

Figure S20. Conversion of 3 to $\mathbf{2}$ via 4 as observed by ${ }^{31} \mathrm{P}$ NMR. A solution of TBDMSCl (6.5 mg, 0.043 $\mathrm{mmol})$ in dry $\mathrm{CH}_{2} \mathrm{Cl}_{2}(0.2 \mathrm{~mL})$ was added to a solution of $\mathbf{3}(16.5 \mathrm{mg}, 0.019 \mathrm{mmol})$ in dry $\mathrm{CH}_{2} \mathrm{Cl}_{2}(0.5$ 
$\mathrm{mL}$ ). The reaction mixture was transferred to an NMR tube and the conversion of $\mathbf{3}$ to $\mathbf{2}$ via $\mathbf{4}$ was monitored by the ${ }^{31} \mathrm{P}$ NMR.

26h

Reaction of 3 and 2 eqv of TBDMSCl

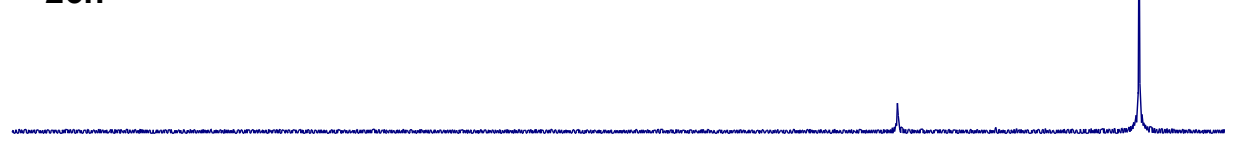

$18 \mathrm{~h}$

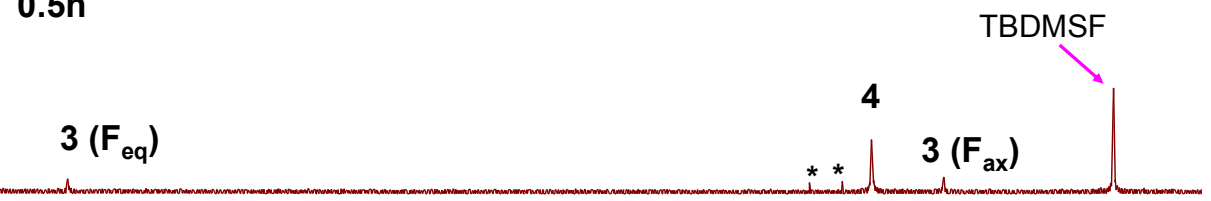

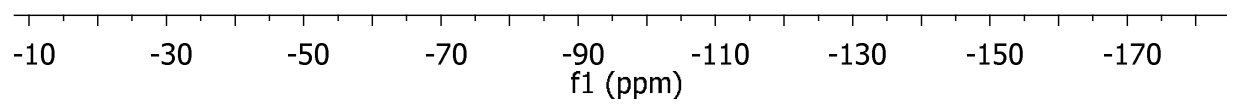

Figure S21. Conversion of $\mathbf{3}$ to $\mathbf{2}$ via $\mathbf{4}$ as observed by ${ }^{19} \mathrm{~F}$ NMR. * denotes impurities. A solution of TBDMSCl $(6.5 \mathrm{mg}, 0.043 \mathrm{mmol})$ in dry $\mathrm{CH}_{2} \mathrm{Cl}_{2}(0.2 \mathrm{~mL})$ was added to a solution of $\mathbf{3}(16.5 \mathrm{mg}, 0.019$ $\mathrm{mmol})$ in dry $\mathrm{CH}_{2} \mathrm{Cl}_{2}(0.5 \mathrm{~mL})$. The reaction mixture was transferred to an NMR tube and the conversion of $\mathbf{3}$ to $\mathbf{2}$ via $\mathbf{4}$ was monitored by the ${ }^{19} \mathrm{~F}$ NMR.
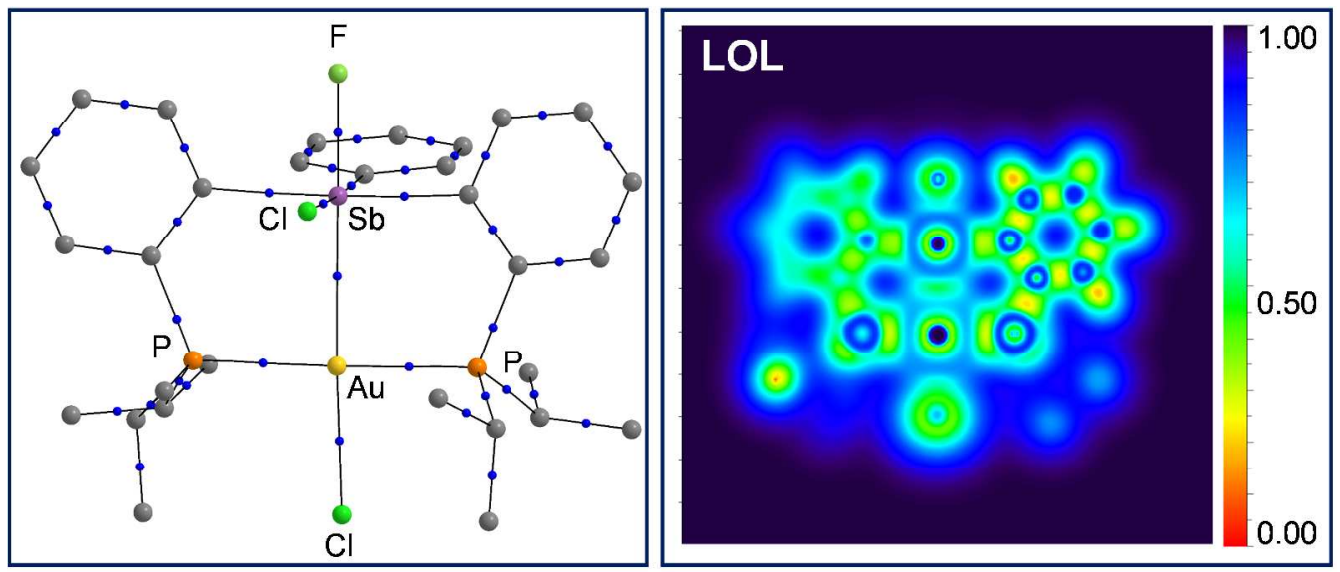

Figure S22. Left: Bond paths and BCPs found for $\mathbf{4}$ using QTAIM. Right: Localized Orbital Locator map of $\mathbf{4}$ in the plane of Sb-Au-P as obtained from Multiwfn software version 3.3.8. 
Table S1. XYZ coordinate of the optimized geometry of complex 2

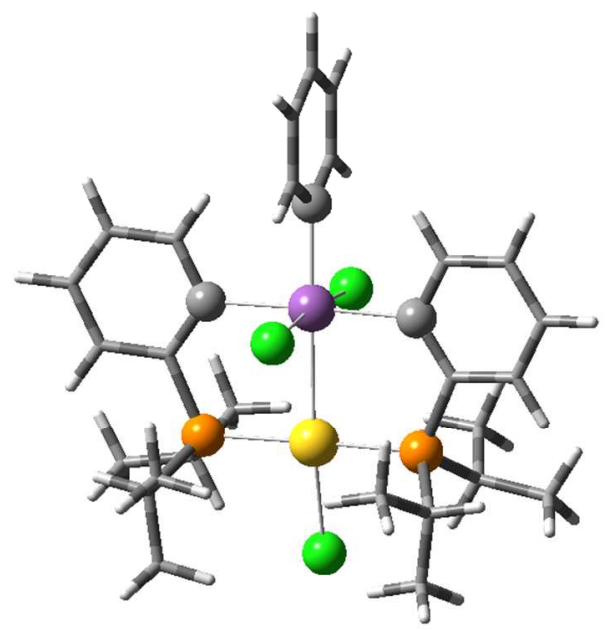

\begin{tabular}{|c|c|c|c|c|c|c|c|}
\hline $\mathrm{Au}$ & 1.412119 & -0.000037 & -0.057538 & $\mathrm{H}$ & 2.182407 & 2.983094 & -3.625201 \\
\hline $\mathrm{Sb}$ & -1.468121 & 0.000048 & 0.003088 & $\mathrm{H}$ & 0.583415 & 3.086565 & -2.828615 \\
\hline $\mathrm{Cl}$ & 3.914635 & -0.000162 & -0.652504 & $\mathrm{H}$ & 1.481732 & 1.545330 & -2.828407 \\
\hline $\mathrm{Cl}$ & -1.227686 & 0.000331 & -2.504468 & $\mathrm{C}$ & 2.309113 & 3.064095 & -1.452822 \\
\hline $\mathrm{Cl}$ & -1.362478 & -0.000190 & 2.517676 & $\mathrm{H}$ & 3.277452 & 2.525202 & -1.401115 \\
\hline $\mathrm{P}$ & 1.413463 & 2.354946 & 0.045414 & $\mathrm{C}$ & 2.561327 & 4.589351 & -1.423401 \\
\hline $\mathrm{P}$ & 1.413364 & -2.355041 & 0.045410 & $\mathrm{H}$ & 3.237811 & 4.854097 & -2.257298 \\
\hline $\mathrm{C}$ & -1.439036 & 2.271728 & 0.034103 & $\mathrm{H}$ & 3.039900 & 4.931035 & -0.488613 \\
\hline $\mathrm{C}$ & -0.271177 & 3.068438 & 0.115006 & $\mathrm{H}$ & 1.624535 & 5.155567 & -1.567870 \\
\hline $\mathrm{C}$ & -0.398644 & 4.479832 & 0.248385 & $\mathrm{C}$ & 3.801386 & 2.829992 & 1.563834 \\
\hline $\mathrm{H}$ & 0.496155 & 5.101213 & 0.344479 & $\mathrm{H}$ & 4.227723 & 3.233050 & 2.501514 \\
\hline $\mathrm{C}$ & -1.659305 & 5.096024 & 0.266177 & $\mathrm{H}$ & 4.287947 & 3.349133 & 0.720477 \\
\hline $\mathrm{H}$ & -1.735579 & 6.182740 & 0.369648 & $\mathrm{H}$ & 4.065242 & 1.761764 & 1.487546 \\
\hline $\mathrm{C}$ & -2.813792 & 4.304182 & 0.149801 & $\mathrm{C}$ & 2.268453 & 3.032185 & 1.582798 \\
\hline $\mathrm{H}$ & -3.806181 & 4.766270 & 0.154024 & $\mathrm{H}$ & 2.050176 & 4.120813 & 1.575552 \\
\hline $\mathrm{C}$ & -2.701980 & 2.904873 & 0.041624 & $\mathrm{C}$ & 1.629856 & 2.414374 & 2.850348 \\
\hline $\mathrm{H}$ & -3.613587 & 2.310486 & -0.027322 & $\mathrm{H}$ & 2.059253 & 2.893870 & 3.749399 \\
\hline $\mathrm{C}$ & -1.439116 & -2.271651 & 0.033768 & $\mathrm{H}$ & 1.838812 & 1.331388 & 2.906447 \\
\hline $\mathrm{C}$ & -0.271331 & -3.068416 & 0.115254 & $\mathrm{H}$ & 0.534811 & 2.542108 & 2.877141 \\
\hline $\mathrm{C}$ & -0.398943 & -4.479757 & 0.249043 & $\mathrm{C}$ & 1.590832 & -2.638871 & -2.756459 \\
\hline $\mathrm{H}$ & 0.495778 & -5.101179 & 0.345603 & $\mathrm{H}$ & 2.182053 & -2.982155 & -3.625462 \\
\hline $\mathrm{C}$ & -1.659649 & -5.095864 & 0.266581 & $\mathrm{H}$ & 1.482830 & -1.544003 & -2.828105 \\
\hline $\mathrm{H}$ & -1.736031 & -6.182536 & 0.370435 & $\mathrm{H}$ & 0.582897 & -3.084298 & -2.829052 \\
\hline $\mathrm{C}$ & -2.814041 & -4.303998 & 0.149409 & $\mathrm{C}$ & 2.308553 & -3.064129 & -1.453116 \\
\hline $\mathrm{H}$ & -3.806457 & -4.766030 & 0.153317 & $\mathrm{H}$ & 3.277338 & -2.526062 & -1.401086 \\
\hline $\mathrm{C}$ & -2.702099 & -2.904729 & 0.040873 & $\mathrm{C}$ & 2.559466 & -4.589599 & -1.424296 \\
\hline $\mathrm{H}$ & -3.613642 & -2.310310 & -0.028641 & $\mathrm{H}$ & 3.235541 & -4.854662 & -2.258420 \\
\hline $\mathrm{C}$ & -3.683695 & 0.000069 & -0.057711 & $\mathrm{H}$ & 1.622145 & -5.154955 & -1.568706 \\
\hline $\mathrm{C}$ & -4.366828 & 0.000314 & -1.297569 & $\mathrm{H}$ & 3.037956 & -4.931971 & -0.489709 \\
\hline $\mathrm{H}$ & -3.799560 & 0.000499 & -2.233169 & $\mathrm{C}$ & 3.801799 & -2.831279 & 1.562694 \\
\hline $\mathrm{C}$ & -5.776827 & 0.000316 & -1.332909 & $\mathrm{H}$ & 4.228325 & -3.234126 & 2.500379 \\
\hline $\mathrm{H}$ & -6.292225 & 0.000503 & -2.299313 & $\mathrm{H}$ & 4.066300 & -1.763257 & 1.485752 \\
\hline $\mathrm{C}$ & -6.518141 & 0.000078 & -0.135800 & $\mathrm{H}$ & 4.287641 & -3.351207 & 0.719396 \\
\hline $\mathrm{H}$ & -7.612710 & 0.000082 & -0.166134 & $\mathrm{C}$ & 2.268767 & -3.032583 & 1.582437 \\
\hline
\end{tabular}




\begin{tabular}{|c|c|c|c|c|c|c|c|}
\hline $\mathrm{C}$ & -5.843925 & -0.000164 & 1.100652 & $\mathrm{H}$ & 2.049926 & -4.121103 & 1.575440 \\
\hline $\mathrm{H}$ & -6.411893 & -0.000346 & 2.037140 & $\mathrm{C}$ & 1.631082 & -2.414250 & 2.850204 \\
\hline $\mathrm{C}$ & -4.434483 & -0.000174 & 1.142759 & $\mathrm{H}$ & 2.060364 & -2.894092 & 3.749122 \\
\hline $\mathrm{H}$ & -3.918277 & -0.000366 & 2.107360 & $\mathrm{H}$ & 0.535935 & -2.541067 & 2.877352 \\
\hline $\mathrm{C}$ & 1.590892 & 2.640055 & -2.756299 & $\mathrm{H}$ & 1.840999 & -1.331441 & 2.906192 \\
\hline
\end{tabular}

Table S2: XYZ coordinates of the optimized geometry of complex 3

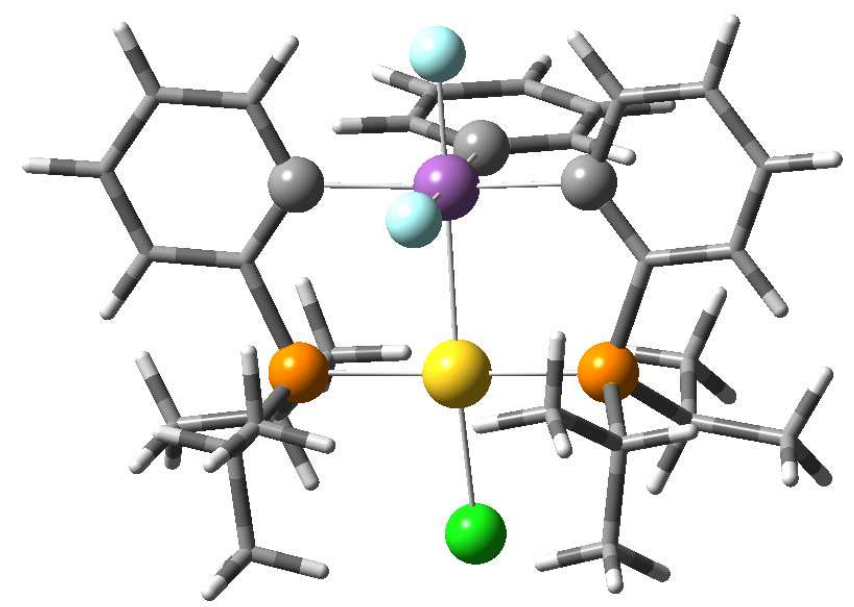

\begin{tabular}{|cccc|cccc|}
\hline $\mathrm{Au}$ & -0.028458 & -1.086189 & 0.202888 & $\mathrm{H}$ & 2.651856 & -2.993614 & -3.266091 \\
$\mathrm{Sb}$ & 0.023483 & 1.542776 & -0.750801 & $\mathrm{H}$ & 2.267573 & -1.278412 & -2.921070 \\
$\mathrm{Cl}$ & -0.070704 & -3.264187 & 1.536368 & $\mathrm{H}$ & 1.129851 & -2.559964 & -2.435383 \\
$\mathrm{P}$ & 2.331253 & -1.134242 & 0.030659 & $\mathrm{C}$ & 2.919018 & -2.471754 & -1.165168 \\
$\mathrm{P}$ & -2.387009 & -1.054740 & -0.005091 & $\mathrm{H}$ & 3.998477 & -2.256023 & -1.310801 \\
$\mathrm{~F}$ & 0.060698 & 3.406035 & -1.511290 & $\mathrm{C}$ & 2.763583 & -3.905860 & -0.609840 \\
$\mathrm{~F}$ & -0.047469 & 0.779258 & -2.637866 & $\mathrm{H}$ & 3.100120 & -4.626387 & -1.378661 \\
$\mathrm{C}$ & 2.228166 & 1.498773 & -1.045024 & $\mathrm{H}$ & 1.713326 & -4.127966 & -0.355313 \\
$\mathrm{C}$ & 3.050017 & 0.402500 & -0.675512 & $\mathrm{H}$ & 3.368452 & -4.075994 & 0.297476 \\
$\mathrm{C}$ & 4.449064 & 0.460930 & -0.930772 & $\mathrm{C}$ & 4.628568 & -1.715963 & 1.709862 \\
$\mathrm{H}$ & 5.092394 & -0.386027 & -0.675242 & $\mathrm{H}$ & 4.939076 & -2.106361 & 2.696884 \\
$\mathrm{C}$ & 5.023268 & 1.594022 & -1.528967 & $\mathrm{H}$ & 5.150771 & -0.755476 & 1.555270 \\
$\mathrm{H}$ & 6.100850 & 1.622114 & -1.718741 & $\mathrm{H}$ & 4.977502 & -2.433075 & 0.945743 \\
$\mathrm{C}$ & 4.205373 & 2.680185 & -1.891630 & $\mathrm{C}$ & 3.094220 & -1.525854 & 1.705220 \\
$\mathrm{H}$ & 4.642834 & 3.563635 & -2.367910 & $\mathrm{H}$ & 2.595810 & -2.486796 & 1.949724 \\
$\mathrm{C}$ & 2.819109 & 2.631481 & -1.654382 & $\mathrm{C}$ & 2.655219 & -0.482091 & 2.758761 \\
$\mathrm{H}$ & 2.179482 & 3.466759 & -1.946334 & $\mathrm{H}$ & 3.038617 & -0.780216 & 3.752037 \\
$\mathrm{C}$ & -2.192680 & 1.625147 & -0.936287 & $\mathrm{H}$ & 1.556658 & -0.410885 & 2.827733 \\
$\mathrm{C}$ & -3.052932 & 0.542758 & -0.617320 & $\mathrm{H}$ & 3.051262 & 0.521941 & 2.526944 \\
$\mathrm{C}$ & -4.452762 & 0.673903 & -0.837870 & $\mathrm{C}$ & -2.313740 & -2.039987 & -2.644791 \\
$\mathrm{H}$ & -5.124785 & -0.163200 & -0.628046 & $\mathrm{H}$ & -2.756685 & -2.705843 & -3.408300 \\
$\mathrm{C}$ & -4.990619 & 1.868074 & -1.344098 & $\mathrm{H}$ & -1.230474 & -2.247030 & -2.596282 \\
$\mathrm{H}$ & -6.069472 & 1.952562 & -1.508164 & $\mathrm{H}$ & -2.433817 & -0.996429 & -2.978845 \\
$\mathrm{C}$ & -4.135578 & 2.943982 & -1.646764 & $\mathrm{C}$ & -2.987809 & -2.309688 & -1.277688 \\
\hline
\end{tabular}




\begin{tabular}{|cccc|cccc|}
\hline $\mathrm{H}$ & -4.545295 & 3.876614 & -2.048020 & $\mathrm{H}$ & -4.075351 & -2.106820 & -1.376869 \\
$\mathrm{C}$ & -2.747647 & 2.821446 & -1.449691 & $\mathrm{C}$ & -2.788227 & -3.773689 & -0.822108 \\
$\mathrm{H}$ & -2.079238 & 3.646080 & -1.705143 & $\mathrm{H}$ & -3.140497 & -4.449962 & -1.623222 \\
$\mathrm{C}$ & 0.105591 & 2.421945 & 1.275807 & $\mathrm{H}$ & -3.352716 & -4.013310 & 0.095332 \\
$\mathrm{C}$ & -1.082909 & 2.659499 & 2.003356 & $\mathrm{H}$ & -1.725730 & -3.992014 & -0.620130 \\
$\mathrm{H}$ & -2.050175 & 2.368706 & 1.581652 & $\mathrm{C}$ & -4.713617 & -1.764687 & 1.598007 \\
$\mathrm{C}$ & -1.040440 & 3.295479 & 3.260778 & $\mathrm{H}$ & -5.029132 & -2.200399 & 2.564362 \\
$\mathrm{H}$ & -1.969970 & 3.476864 & 3.811181 & $\mathrm{H}$ & -5.018580 & -2.468125 & 0.803515 \\
$\mathrm{C}$ & 0.192418 & 3.706697 & 3.802340 & $\mathrm{H}$ & -5.271005 & -0.820681 & 1.465688 \\
$\mathrm{H}$ & 0.226088 & 4.203723 & 4.777362 & $\mathrm{C}$ & -3.187598 & -1.517616 & 1.635935 \\
$\mathrm{C}$ & 1.380881 & 3.487306 & 3.079387 & $\mathrm{H}$ & -2.661572 & -2.466045 & 1.871510 \\
$\mathrm{H}$ & 2.341200 & 3.820751 & 3.487490 & $\mathrm{C}$ & -2.816241 & -0.487665 & 2.727810 \\
$\mathrm{C}$ & 1.337887 & 2.847950 & 1.823941 & $\mathrm{H}$ & -3.178724 & -0.845863 & 3.708903 \\
$\mathrm{H}$ & 2.267078 & 2.703844 & 1.263608 & $\mathrm{H}$ & -3.283367 & 0.493343 & 2.529834 \\
$\mathrm{C}$ & 2.200751 & -2.306202 & -2.526968 & $\mathrm{H}$ & -1.724907 & -0.344479 & 2.801701 \\
& & & & & & \\
\hline
\end{tabular}

Table S3: XYZ coordinates of the optimized geometry of complex 4

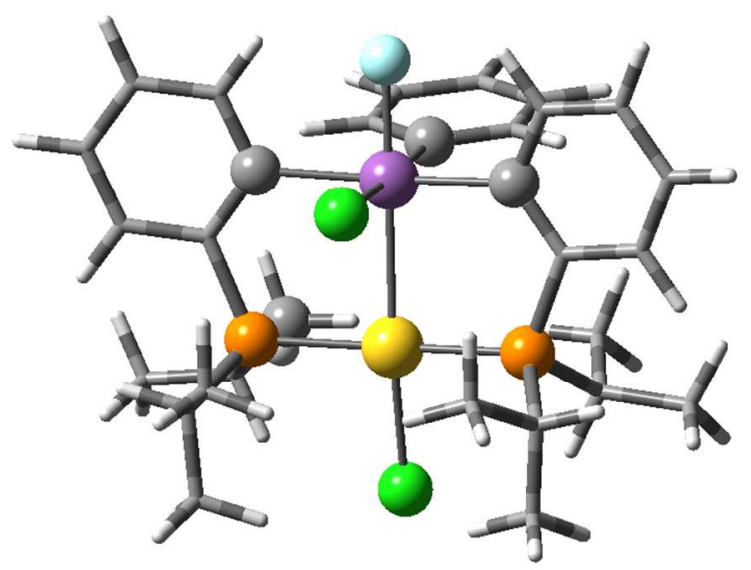

\begin{tabular}{|cccc|crrr|}
\hline $\mathrm{Au}$ & -0.000007 & 1.106114 & 0.220307 & $\mathrm{H}$ & -2.170889 & -2.490189 & 1.542325 \\
$\mathrm{Sb}$ & -0.000047 & -1.548455 & -0.653174 & $\mathrm{C}$ & -3.124514 & 1.545299 & 1.726021 \\
$\mathrm{P}$ & 2.363861 & 1.108370 & 0.059472 & $\mathrm{H}$ & -2.644806 & 2.525043 & 1.929886 \\
$\mathrm{P}$ & -2.363779 & 1.108499 & 0.059387 & $\mathrm{C}$ & 1.216737 & -2.697858 & 2.036509 \\
$\mathrm{Cl}$ & -0.000061 & -0.733380 & -3.086667 & $\mathrm{H}$ & 2.170846 & -2.490345 & 1.542108 \\
$\mathrm{C}$ & 2.215521 & -1.576030 & -0.885290 & $\mathrm{C}$ & 2.994103 & 2.394483 & -1.170017 \\
$\mathrm{~F}$ & -0.000118 & -3.442258 & -1.342519 & $\mathrm{H}$ & 4.067515 & 2.143769 & -1.303328 \\
$\mathrm{C}$ & 3.051589 & -0.474457 & -0.571064 & $\mathrm{C}$ & -1.217411 & -3.309836 & 3.306250 \\
$\mathrm{C}$ & -2.215627 & -1.575970 & -0.885176 & $\mathrm{H}$ & -2.168203 & -3.557582 & 3.790473 \\
$\mathrm{C}$ & -3.051630 & -0.474386 & -0.570819 & $\mathrm{C}$ & 2.277573 & 2.217551 & -2.530386 \\
$\mathrm{C}$ & 5.007311 & -1.757122 & -1.330193 & $\mathrm{H}$ & 1.221772 & 2.532736 & -2.457570 \\
$\mathrm{H}$ & 6.085384 & -1.817326 & -1.508805 & $\mathrm{H}$ & 2.282478 & 1.173856 & -2.886249 \\
$\mathrm{C}$ & 3.124671 & 1.544881 & 1.726175 & $\mathrm{H}$ & 2.771368 & 2.851166 & -3.290024 \\
$\mathrm{H}$ & 2.645474 & 2.524901 & 1.929900 & $\mathrm{C}$ & -2.993938 & 2.394439 & -1.170311 \\
$\mathrm{C}$ & 4.451021 & -0.577013 & -0.811422 & $\mathrm{H}$ & -4.067504 & 2.144079 & -1.303120 \\
$\mathrm{H}$ & 5.109016 & 0.271652 & -0.603902 & $\mathrm{C}$ & -2.663764 & 0.555992 & 2.820826 \\
\hline
\end{tabular}




\begin{tabular}{|cccl|rrrr|}
\hline $\mathrm{C}$ & -4.451115 & -0.576960 & -0.810875 & $\mathrm{H}$ & -3.030796 & 0.901999 & 3.804706 \\
$\mathrm{H}$ & -5.109061 & 0.271723 & -0.603288 & $\mathrm{H}$ & -1.564190 & 0.489299 & 2.874346 \\
$\mathrm{C}$ & -5.007507 & -1.757095 & -1.329477 & $\mathrm{H}$ & -3.063388 & -0.457862 & 2.645781 \\
$\mathrm{H}$ & -6.085623 & -1.817321 & -1.507831 & $\mathrm{C}$ & 2.876678 & 3.846469 & -0.651878 \\
$\mathrm{C}$ & 4.662884 & 1.704118 & 1.730868 & $\mathrm{H}$ & 3.227866 & 4.536425 & -1.441767 \\
$\mathrm{H}$ & 4.976836 & 2.124875 & 2.704237 & $\mathrm{H}$ & 3.488519 & 4.027524 & 0.248541 \\
$\mathrm{H}$ & 5.030310 & 2.384548 & 0.942433 & $\mathrm{H}$ & 1.832389 & 4.100797 & -0.402627 \\
$\mathrm{H}$ & 5.166033 & 0.728114 & 1.614786 & $\mathrm{C}$ & -4.662647 & 1.705335 & 1.730789 \\
$\mathrm{C}$ & -4.172361 & -2.849968 & -1.626751 & $\mathrm{H}$ & -5.166319 & 0.729584 & 1.614842 \\
$\mathrm{H}$ & -4.596333 & -3.771504 & -2.038394 & $\mathrm{H}$ & -5.029781 & 2.385897 & 0.942340 \\
$\mathrm{C}$ & 2.663458 & 0.555965 & 2.821106 & $\mathrm{H}$ & -4.976275 & 2.126324 & 2.704157 \\
$\mathrm{H}$ & 3.063129 & -0.457951 & 2.646534 & $\mathrm{C}$ & -2.278007 & 2.216856 & -2.530888 \\
$\mathrm{H}$ & 1.563864 & 0.489289 & 2.874242 & $\mathrm{H}$ & -2.771553 & 2.850825 & -3.290392 \\
$\mathrm{H}$ & 3.030088 & 0.902306 & 3.805015 & $\mathrm{H}$ & -2.283903 & 1.173137 & -2.886651 \\
$\mathrm{C}$ & 4.172112 & -2.849997 & -1.627302 & $\mathrm{H}$ & -1.221914 & 2.531130 & -2.458494 \\
$\mathrm{H}$ & 4.596007 & -3.771513 & -2.039069 & $\mathrm{C}$ & -2.875778 & 3.846521 & -0.652611 \\
$\mathrm{C}$ & 2.784730 & -2.759032 & -1.410904 & $\mathrm{H}$ & -1.831260 & 4.100629 & -0.404106 \\
$\mathrm{H}$ & 2.130031 & -3.596040 & -1.661197 & $\mathrm{H}$ & -3.486924 & 4.027986 & 0.248190 \\
$\mathrm{C}$ & -2.784930 & -2.758982 & -1.410656 & $\mathrm{H}$ & -3.227300 & 4.536369 & -1.442445 \\
$\mathrm{H}$ & -2.130274 & -3.595987 & -1.661067 & $\mathrm{C}$ & 0.000054 & -3.612177 & 3.945640 \\
$\mathrm{Cl}$ & 0.000194 & 3.320905 & 1.478845 & $\mathrm{H}$ & 0.000086 & -4.089027 & 4.931235 \\
$\mathrm{C}$ & -0.000024 & -2.378001 & 1.389813 & $\mathrm{C}$ & 1.217479 & -3.309942 & 3.306124 \\
$\mathrm{C}$ & -1.216747 & -2.697761 & 2.036630 & $\mathrm{H}$ & 2.168300 & -3.557783 & 3.790244 \\
& & & & & & & \\
& & & & & & & \\
\hline
\end{tabular}

Table S4: XYZ coordinates of the optimized geometry of complex 5 ( $\mathrm{PF}_{6}$ unit has been omitted)

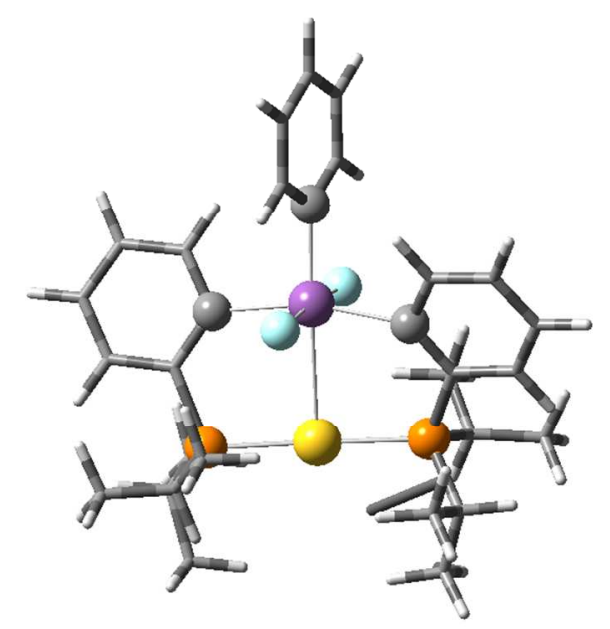

\begin{tabular}{|c|c|c|c|c|c|c|c|}
\hline $\mathrm{Au}$ & -0.682833 & 0.157658 & 0.513813 & $\mathrm{H}$ & -2.765706 & -2.334960 & -1.042171 \\
\hline $\mathrm{Sb}$ & 2.288678 & -0.103791 & -0.170494 & $\mathrm{C}$ & -2.026869 & -4.380230 & -1.049419 \\
\hline $\mathrm{P}$ & -0.905114 & -2.176756 & 0.427419 & $\mathrm{H}$ & -2.661101 & -4.650027 & -1.913015 \\
\hline $\mathrm{P}$ & -0.406595 & 2.467974 & 0.782652 & $\mathrm{H}$ & -2.558909 & -4.712282 & -0.140432 \\
\hline $\mathrm{F}$ & 1.552633 & -0.545409 & -1.987361 & $\mathrm{H}$ & -1.081340 & -4.944696 & -1.140976 \\
\hline
\end{tabular}




\begin{tabular}{|c|c|c|c|c|c|c|c|}
\hline $\mathrm{F}$ & 2.834004 & 0.381118 & 1.710601 & $\mathrm{C}$ & -3.282627 & -2.235295 & 1.959861 \\
\hline $\mathrm{C}$ & 1.973708 & -2.189707 & 0.498128 & $\mathrm{H}$ & -3.814196 & -2.641507 & 2.839976 \\
\hline $\mathrm{C}$ & 0.764318 & -2.931503 & 0.583930 & $\mathrm{H}$ & -3.856616 & -2.503824 & 1.059272 \\
\hline $\mathrm{C}$ & 0.841381 & -4.314040 & 0.910175 & $\mathrm{H}$ & -3.300308 & -1.133577 & 2.032606 \\
\hline $\mathrm{H}$ & -0.078189 & -4.901563 & 0.981343 & $\mathrm{C}$ & -1.833346 & -2.778771 & 1.951552 \\
\hline $\mathrm{C}$ & 2.071084 & -4.941880 & 1.165395 & $\mathrm{H}$ & -1.866721 & -3.885103 & 1.877409 \\
\hline $\mathrm{H}$ & 2.098206 & -6.006844 & 1.414171 & $\mathrm{C}$ & -1.074461 & -2.389248 & 3.242847 \\
\hline $\mathrm{C}$ & 3.255603 & -4.189248 & 1.116902 & $\mathrm{H}$ & -1.636180 & -2.756553 & 4.121015 \\
\hline $\mathrm{H}$ & 4.220993 & -4.655123 & 1.335978 & $\mathrm{H}$ & -0.990833 & -1.290274 & 3.332280 \\
\hline $\mathrm{C}$ & 3.205298 & -2.823046 & 0.782039 & $\mathrm{H}$ & -0.057680 & -2.815280 & 3.281502 \\
\hline $\mathrm{H}$ & 4.137896 & -2.256342 & 0.740400 & $\mathrm{C}$ & -1.961254 & 3.137077 & -1.473020 \\
\hline $\mathrm{C}$ & 2.077002 & 2.062225 & -0.599227 & $\mathrm{H}$ & -2.755368 & 3.772865 & -1.902236 \\
\hline $\mathrm{C}$ & 1.110847 & 2.985923 & -0.111189 & $\mathrm{H}$ & -2.272282 & 2.090253 & -1.624047 \\
\hline $\mathrm{C}$ & 1.269059 & 4.363445 & -0.426025 & $\mathrm{H}$ & -1.028444 & 3.322999 & -2.033180 \\
\hline $\mathrm{H}$ & 0.532762 & 5.088061 & -0.067490 & $\mathrm{C}$ & -1.813466 & 3.461690 & 0.033670 \\
\hline $\mathrm{C}$ & 2.337371 & 4.818912 & -1.215095 & $\mathrm{H}$ & -1.537665 & 4.530722 & 0.148408 \\
\hline $\mathrm{H}$ & 2.431020 & 5.884577 & -1.443445 & $\mathrm{C}$ & -3.126201 & 3.197933 & 0.813527 \\
\hline $\mathrm{C}$ & 3.263232 & 3.894773 & -1.724071 & $\mathrm{H}$ & -3.950077 & 3.746473 & 0.325295 \\
\hline $\mathrm{H}$ & 4.086599 & 4.226097 & -2.363810 & $\mathrm{H}$ & -3.069659 & 3.535993 & 1.863579 \\
\hline $\mathrm{C}$ & 3.133994 & 2.527582 & -1.413630 & $\mathrm{H}$ & -3.404419 & 2.131068 & 0.786576 \\
\hline $\mathrm{H}$ & 3.867247 & 1.825552 & -1.815347 & $\mathrm{C}$ & -0.130534 & 4.533377 & 2.765773 \\
\hline $\mathrm{C}$ & 4.333135 & -0.332782 & -0.816568 & $\mathrm{H}$ & -0.162316 & 4.777111 & 3.843563 \\
\hline $\mathrm{C}$ & 5.392056 & 0.051101 & 0.040261 & $\mathrm{H}$ & -0.934652 & 5.106844 & 2.272524 \\
\hline $\mathrm{H}$ & 5.170793 & 0.468100 & 1.027366 & $\mathrm{H}$ & 0.842731 & 4.880164 & 2.376037 \\
\hline $\mathrm{C}$ & 6.726877 & -0.108703 & -0.382522 & $\mathrm{C}$ & -0.288981 & 3.004914 & 2.587397 \\
\hline $\mathrm{H}$ & 7.545393 & 0.187827 & 0.281290 & $\mathrm{H}$ & -1.275841 & 2.699496 & 2.993293 \\
\hline $\mathrm{C}$ & 7.007565 & -0.647422 & -1.653873 & $\mathrm{C}$ & 0.815767 & 2.230446 & 3.340701 \\
\hline $\mathrm{H}$ & 8.045535 & -0.769626 & -1.979311 & $\mathrm{H}$ & 0.790900 & 2.507915 & 4.410635 \\
\hline $\mathrm{C}$ & 5.952790 & -1.028115 & -2.506760 & $\mathrm{H}$ & 1.817880 & 2.465179 & 2.945225 \\
\hline $\mathrm{H}$ & 6.169453 & -1.445665 & -3.495204 & $\mathrm{H}$ & 0.684844 & 1.138456 & 3.262731 \\
\hline $\mathrm{C}$ & 4.614157 & -0.873536 & -2.092959 & $\mathrm{P}$ & -4.980863 & -0.075951 & -1.207727 \\
\hline $\mathrm{H}$ & 3.794348 & -1.165343 & -2.755828 & $\mathrm{~F}$ & -4.992347 & 1.567146 & -1.423486 \\
\hline $\mathrm{C}$ & -1.063058 & -2.419525 & -2.380434 & $\mathrm{~F}$ & -3.358018 & 0.033248 & -0.665910 \\
\hline $\mathrm{H}$ & -1.670746 & -2.736518 & -3.246532 & $\mathrm{~F}$ & -4.894903 & -1.731631 & -0.965113 \\
\hline $\mathrm{H}$ & -0.063706 & -2.881455 & -2.465527 & $\mathrm{~F}$ & -6.536035 & -0.202988 & -1.712153 \\
\hline $\mathrm{H}$ & -0.937688 & -1.326819 & -2.438977 & $\mathrm{~F}$ & -5.464990 & 0.121012 & 0.368283 \\
\hline $\mathrm{C}$ & -1.787399 & -2.852534 & -1.084234 & $\mathrm{~F}$ & -4.411279 & -0.285377 & -2.746524 \\
\hline
\end{tabular}


Table S5: XYZ coordinates of the optimized geometry of complex 6

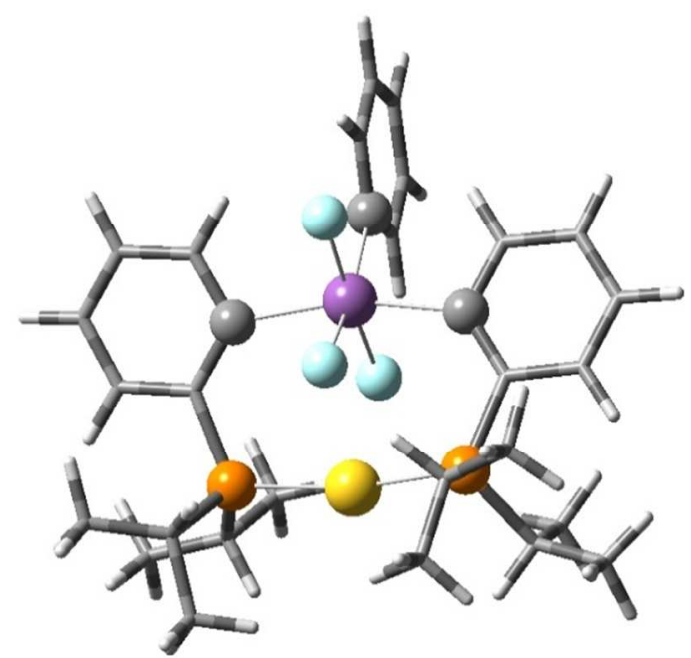

\begin{tabular}{|c|c|c|c|c|c|c|c|}
\hline $\mathrm{P}$ & 1.239314 & -2.468937 & 0.208951 & $\mathrm{H}$ & -3.911470 & -1.660499 & 1.449665 \\
\hline $\mathrm{P}$ & -2.794189 & -0.160746 & 0.016166 & $\mathrm{H}$ & -5.938546 & -0.660527 & 0.272907 \\
\hline $\mathrm{C}$ & 2.462214 & 0.010507 & -0.775524 & $\mathrm{H}$ & -6.093953 & -0.755060 & 2.045617 \\
\hline $\mathrm{C}$ & 3.590288 & 0.682528 & -1.306774 & $\mathrm{H}$ & -5.770295 & 0.810739 & 1.276972 \\
\hline $\mathrm{H}$ & 3.490570 & 1.728088 & -1.609698 & $\mathrm{H}$ & -2.435167 & -0.240717 & 2.899661 \\
\hline $\mathrm{C}$ & 4.837942 & 0.051166 & -1.451824 & $\mathrm{H}$ & -3.564613 & 1.131758 & 2.739287 \\
\hline $\mathrm{H}$ & 5.684578 & 0.605855 & -1.868540 & $\mathrm{H}$ & -4.088289 & -0.358959 & 3.579850 \\
\hline $\mathrm{C}$ & 4.987218 & -1.285308 & -1.049926 & $\mathrm{C}$ & -3.514717 & -0.622812 & -1.668826 \\
\hline $\mathrm{H}$ & 5.953354 & -1.792234 & -1.137369 & $\mathrm{C}$ & -4.604734 & 0.311614 & -2.240307 \\
\hline $\mathrm{C}$ & 3.877929 & -1.978444 & -0.538771 & $\mathrm{C}$ & -3.957586 & -2.107111 & -1.670195 \\
\hline $\mathrm{H}$ & 4.007776 & -3.023471 & -0.250820 & $\mathrm{H}$ & -2.610074 & -0.525444 & -2.303508 \\
\hline $\mathrm{C}$ & 2.598992 & -1.364193 & -0.402917 & $\mathrm{H}$ & -4.263434 & 1.358601 & -2.285218 \\
\hline $\mathrm{C}$ & -1.240798 & 2.295075 & -0.312866 & $\mathrm{H}$ & -4.845748 & -0.008386 & -3.271521 \\
\hline $\mathrm{C}$ & -1.192140 & 3.706031 & -0.409120 & $\mathrm{H}$ & -5.540519 & 0.275538 & -1.655003 \\
\hline $\mathrm{H}$ & -0.233704 & 4.193516 & -0.608252 & $\mathrm{H}$ & -3.155828 & -2.766735 & -1.290985 \\
\hline $\mathrm{C}$ & -2.337186 & 4.506039 & -0.245194 & $\mathrm{H}$ & -4.860686 & -2.267827 & -1.052926 \\
\hline $\mathrm{H}$ & -2.257957 & 5.594717 & -0.326633 & $\mathrm{H}$ & -4.199836 & -2.420539 & -2.702329 \\
\hline $\mathrm{C}$ & -3.572516 & 3.897498 & 0.024746 & $\mathrm{C}$ & 1.741959 & -3.169102 & 1.894335 \\
\hline $\mathrm{H}$ & -4.476244 & 4.500068 & 0.159774 & $\mathrm{C}$ & 1.906483 & -1.994035 & 2.891793 \\
\hline $\mathrm{C}$ & -3.646223 & 2.497389 & 0.113086 & $\mathrm{C}$ & 2.947962 & -4.133866 & 1.971796 \\
\hline $\mathrm{H}$ & -4.619079 & 2.039254 & 0.301352 & $\mathrm{H}$ & 0.827666 & -3.733072 & 2.173892 \\
\hline $\mathrm{C}$ & -2.500313 & 1.669707 & -0.053366 & $\mathrm{H}$ & 1.043661 & -1.307576 & 2.867502 \\
\hline $\mathrm{Au}$ & -0.847909 & -1.421410 & 0.314640 & $\mathrm{H}$ & 2.019067 & -2.392944 & 3.917172 \\
\hline $\mathrm{F}$ & -0.219474 & 0.061829 & -1.807873 & $\mathrm{H}$ & 2.806302 & -1.400059 & 2.652889 \\
\hline $\mathrm{C}$ & 1.922615 & 3.042979 & 0.350761 & $\mathrm{H}$ & 2.873092 & -4.974668 & 1.261286 \\
\hline $\mathrm{C}$ & 2.483989 & 4.079496 & -0.435735 & $\mathrm{H}$ & 3.899468 & -3.604196 & 1.795257 \\
\hline $\mathrm{C}$ & 2.126718 & 3.056289 & 1.751036 & $\mathrm{H}$ & 2.998724 & -4.561933 & 2.990978 \\
\hline $\mathrm{C}$ & 3.236530 & 5.106677 & 0.170003 & $\mathrm{C}$ & 1.047455 & -3.881853 & -1.046153 \\
\hline $\mathrm{H}$ & 2.327750 & 4.078775 & -1.518205 & $\mathrm{C}$ & 0.110050 & -4.963311 & -0.447288 \\
\hline $\mathrm{C}$ & 2.880507 & 4.085275 & 2.351281 & $\mathrm{C}$ & 2.301696 & -4.522385 & -1.682309 \\
\hline $\mathrm{H}$ & 1.694083 & 2.262025 & 2.365174 & $\mathrm{H}$ & 0.494327 & -3.358365 & -1.853448 \\
\hline
\end{tabular}




\begin{tabular}{|lccc|cccc|}
\hline $\mathrm{C}$ & 3.436991 & 5.111568 & 1.563710 & $\mathrm{H}$ & -0.810145 & -4.519449 & -0.025582 \\
$\mathrm{H}$ & 3.665066 & 5.902470 & -0.449452 & $\mathrm{H}$ & -0.185907 & -5.675292 & -1.239584 \\
$\mathrm{H}$ & 3.031708 & 4.084019 & 3.436529 & $\mathrm{H}$ & 0.614608 & -5.542073 & 0.348229 \\
$\mathrm{H}$ & 4.022061 & 5.909943 & 2.032855 & $\mathrm{H}$ & 2.935675 & -3.778021 & -2.189346 \\
$\mathrm{~F}$ & 1.108817 & 2.322314 & -2.324946 & $\mathrm{H}$ & 2.918107 & -5.065254 & -0.943926 \\
$\mathrm{C}$ & -4.029323 & -0.557374 & 1.402659 & $\mathrm{H}$ & 1.974586 & -5.258543 & -2.441169 \\
$\mathrm{C}$ & -5.538908 & -0.266535 & 1.221900 & $\mathrm{Sb}$ & 0.765209 & 1.410204 & -0.566688 \\
$\mathrm{C}$ & -3.492815 & 0.029989 & 2.733175 & $\mathrm{~F}$ & 0.515090 & 0.641606 & 1.344520 \\
& & & & & & \\
\hline
\end{tabular}

Table S6: XYZ coordinates of the optimized geometry of complex 7

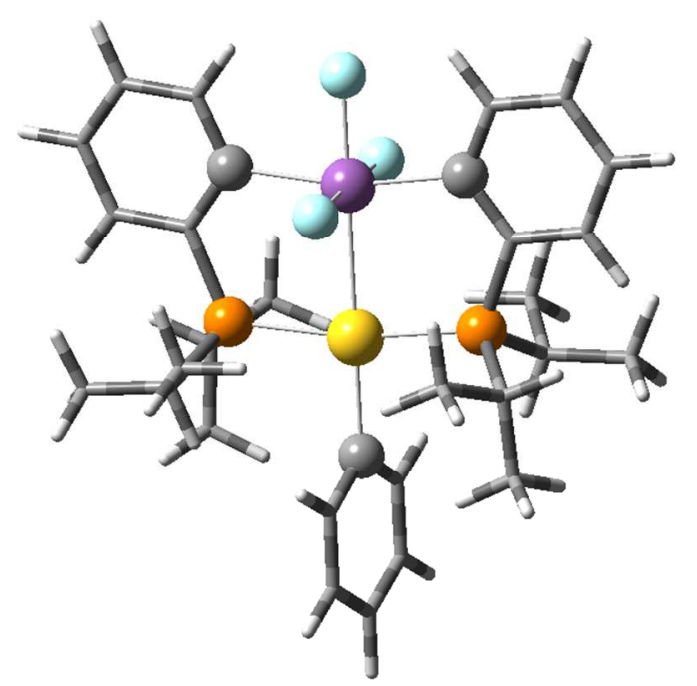

\begin{tabular}{|cccc|crrr|}
\hline $\mathrm{Au}$ & -0.000012 & 0.692687 & 0.000055 & $\mathrm{H}$ & -2.746882 & 1.160256 & -3.664115 \\
$\mathrm{Sb}$ & 0.000162 & -2.106237 & -0.000002 & $\mathrm{H}$ & -2.749268 & -0.427165 & -2.837961 \\
$\mathrm{P}$ & -2.368776 & 0.486537 & 0.054460 & $\mathrm{H}$ & -1.320338 & 0.618221 & -2.724305 \\
$\mathrm{~F}$ & 0.001587 & -2.047091 & 2.030278 & $\mathrm{C}$ & -3.044039 & 1.303725 & -1.505296 \\
$\mathrm{C}$ & -2.194482 & -2.357854 & 0.027659 & $\mathrm{H}$ & -2.652715 & 2.338416 & -1.428739 \\
$\mathrm{P}$ & 2.368717 & 0.486810 & -0.054389 & $\mathrm{C}$ & -4.585199 & 1.359682 & -1.622202 \\
$\mathrm{~F}$ & -0.001207 & -2.046777 & -2.030309 & $\mathrm{H}$ & -4.855338 & 1.981839 & -2.495988 \\
$\mathrm{C}$ & -3.039085 & -1.228555 & 0.102268 & $\mathrm{H}$ & -5.071670 & 1.803688 & -0.735586 \\
$\mathrm{~F}$ & 0.000233 & -4.115647 & -0.000093 & $\mathrm{H}$ & -5.007816 & 0.353928 & -1.787900 \\
$\mathrm{C}$ & -4.446024 & -1.415022 & 0.217696 & $\mathrm{C}$ & -3.271505 & 2.838529 & 1.474889 \\
$\mathrm{H}$ & -5.116731 & -0.555050 & 0.303362 & $\mathrm{H}$ & -3.791968 & 3.211777 & 2.376884 \\
$\mathrm{C}$ & -4.997001 & -2.706147 & 0.230003 & $\mathrm{H}$ & -3.838894 & 3.185512 & 0.594305 \\
$\mathrm{H}$ & -6.080449 & -2.833480 & 0.319991 & $\mathrm{H}$ & -2.272140 & 3.300670 & 1.442244 \\
$\mathrm{C}$ & -4.151312 & -3.826756 & 0.130495 & $\mathrm{C}$ & -3.178050 & 1.298804 & 1.552056 \\
$\mathrm{H}$ & -4.573531 & -4.836876 & 0.138801 & $\mathrm{H}$ & -4.205153 & 0.880696 & 1.570531 \\
$\mathrm{C}$ & -2.758613 & -3.654854 & 0.036123 & $\mathrm{C}$ & -2.445302 & 0.827304 & 2.833599 \\
$\mathrm{H}$ & -2.094313 & -4.517670 & -0.018474 & $\mathrm{H}$ & -2.997954 & 1.174344 & 3.726076 \\
$\mathrm{C}$ & 2.194829 & -2.357609 & -0.027699 & $\mathrm{H}$ & -1.425660 & 1.250007 & 2.878313 \\
$\mathrm{C}$ & 3.039279 & -1.228183 & -0.102218 & $\mathrm{H}$ & -2.355779 & -0.271069 & 2.884893 \\
$\mathrm{C}$ & 4.446247 & -1.414431 & -0.217624 & $\mathrm{C}$ & 2.444485 & 0.827719 & -2.833475 \\
\hline
\end{tabular}




\begin{tabular}{|cccc|cccc|}
\hline $\mathrm{H}$ & 5.116823 & -0.554354 & -0.303241 & $\mathrm{H}$ & 2.996965 & 1.174602 & -3.726119 \\
$\mathrm{C}$ & 4.997415 & -2.705476 & -0.229982 & $\mathrm{H}$ & 1.424926 & 1.250643 & -2.877897 \\
$\mathrm{H}$ & 6.080884 & -2.832645 & -0.319949 & $\mathrm{H}$ & 2.354687 & -0.270638 & -2.884703 \\
$\mathrm{C}$ & 4.151883 & -3.826212 & -0.130568 & $\mathrm{C}$ & 3.177670 & 1.299122 & -1.552144 \\
$\mathrm{H}$ & 4.574249 & -4.836270 & -0.138908 & $\mathrm{H}$ & 4.204746 & 0.880963 & -1.570921 \\
$\mathrm{C}$ & 2.759156 & -3.654525 & -0.036233 & $\mathrm{C}$ & 3.271194 & 2.838838 & -1.474927 \\
$\mathrm{H}$ & 2.094989 & -4.517448 & 0.018295 & $\mathrm{H}$ & 3.791092 & 3.212145 & -2.377223 \\
$\mathrm{C}$ & -0.000208 & 2.872577 & -0.000036 & $\mathrm{H}$ & 3.839155 & 3.185727 & -0.594674 \\
$\mathrm{C}$ & 0.272699 & 3.615736 & 1.180187 & $\mathrm{H}$ & 2.271853 & 3.300988 & -1.441639 \\
$\mathrm{H}$ & 0.472715 & 3.091385 & 2.122688 & $\mathrm{C}$ & 4.585117 & 1.360272 & 1.622161 \\
$\mathrm{C}$ & 0.275471 & 5.027741 & 1.183299 & $\mathrm{H}$ & 4.855203 & 1.982444 & 2.495946 \\
$\mathrm{H}$ & 0.489340 & 5.569436 & 2.111920 & $\mathrm{H}$ & 5.071569 & 1.804314 & 0.735548 \\
$\mathrm{C}$ & -0.000433 & 5.739216 & -0.000119 & $\mathrm{H}$ & 5.007803 & 0.354549 & 1.787868 \\
$\mathrm{H}$ & -0.000516 & 6.834023 & -0.000150 & $\mathrm{C}$ & 3.043964 & 1.304222 & 1.505244 \\
$\mathrm{C}$ & -0.276203 & 5.027630 & -1.183498 & $\mathrm{H}$ & 2.652591 & 2.338884 & 1.428526 \\
$\mathrm{H}$ & -0.490144 & 5.569232 & -2.112156 & $\mathrm{C}$ & 2.423824 & 0.624820 & 2.752478 \\
$\mathrm{C}$ & -0.273197 & 3.615625 & -1.180306 & $\mathrm{H}$ & 2.746773 & 1.161095 & 3.664084 \\
$\mathrm{H}$ & -0.473074 & 3.091197 & -2.122794 & $\mathrm{H}$ & 2.749367 & -0.426464 & 2.838195 \\
$\mathrm{C}$ & -2.423850 & 0.624172 & -2.752425 & $\mathrm{H}$ & 1.320307 & 0.618743 & 2.724351 \\
& & & & & & \\
\hline
\end{tabular}

Table S7. Selected bond lengths $(\AA)$ and angles $\left({ }^{\circ}\right)$ for complexes $2,3,4,5$ and 7 as determined crystallographically and optimized computationally. (* denotes the calculated parameters)

\begin{tabular}{|c|c|c|c|c|}
\hline & Sb-Au $(\AA)$ & P-Au-P $\left(^{0}\right)$ & Sb-Au-X $\left(^{0}\right)$ & $\Sigma_{\alpha} \mathrm{Au}\left(\mathbf{(}^{0}\right)$ \\
\hline 2 & $2.8651(4)$ & $166.03(3)$ & $160.217(18)$ & 364.89 \\
\hline $2 *$ & 2.8808 & 174.993 & 167.832 & 361.054 \\
\hline 3 & $2.7450(14)$ & $163.82(5)$ & $164.18(3)$ & 364.13 \\
\hline $3 *$ & 2.7971 & 170.779 & 168.463 & 361.703 \\
\hline 4 & $2.7327(9)$ & $165.37(5)$ & $164.65(3)$ & 363.64 \\
\hline $4 *$ & 2.7946 & 172.212 & 168.607 & 361.422 \\
\hline 5 & $3.0708(13)$ & $178.70(11)$ & - & - \\
\hline 5 & 3.0608 & 175.313 & - & - \\
\hline 7 & $2.6886(5)$ & $171.84(5)$ & $177.14(19)$ & 360.17 \\
\hline $7 *$ & 2.7989 & 170.061 & 179.996 & 359.99 \\
\hline
\end{tabular}


Table S8. Selected bond lengths $(\AA)$ and angles $\left(^{\circ}\right)$ for complex 6 as determined crystallographically and optimized computationally.

\begin{tabular}{|c|c|c|c|c|c|c|c|}
\hline & Sb---Au (Å) & Au---F1 (Å) & Au---F2(A) & Sb-F1 (̊̊) & Sb-F2 (Å) & Sb-F3(Å) & $\begin{array}{l}\text { P-Au-P } \\
\left({ }^{0}\right)\end{array}$ \\
\hline 6 & $3.3918(4)$ & $2.6644(32)$ & $2.6755(28)$ & $2.010(3)$ & $2.009(3)$ & $1.973(3)$ & $168.19(5)$ \\
\hline 6* & 3.37593 & 2.6646 & 2.6785 & 2.0845 & 2.0751 & 2.010 & 168.261 \\
\hline
\end{tabular}

Mogul search - Bond length - Au7 C6

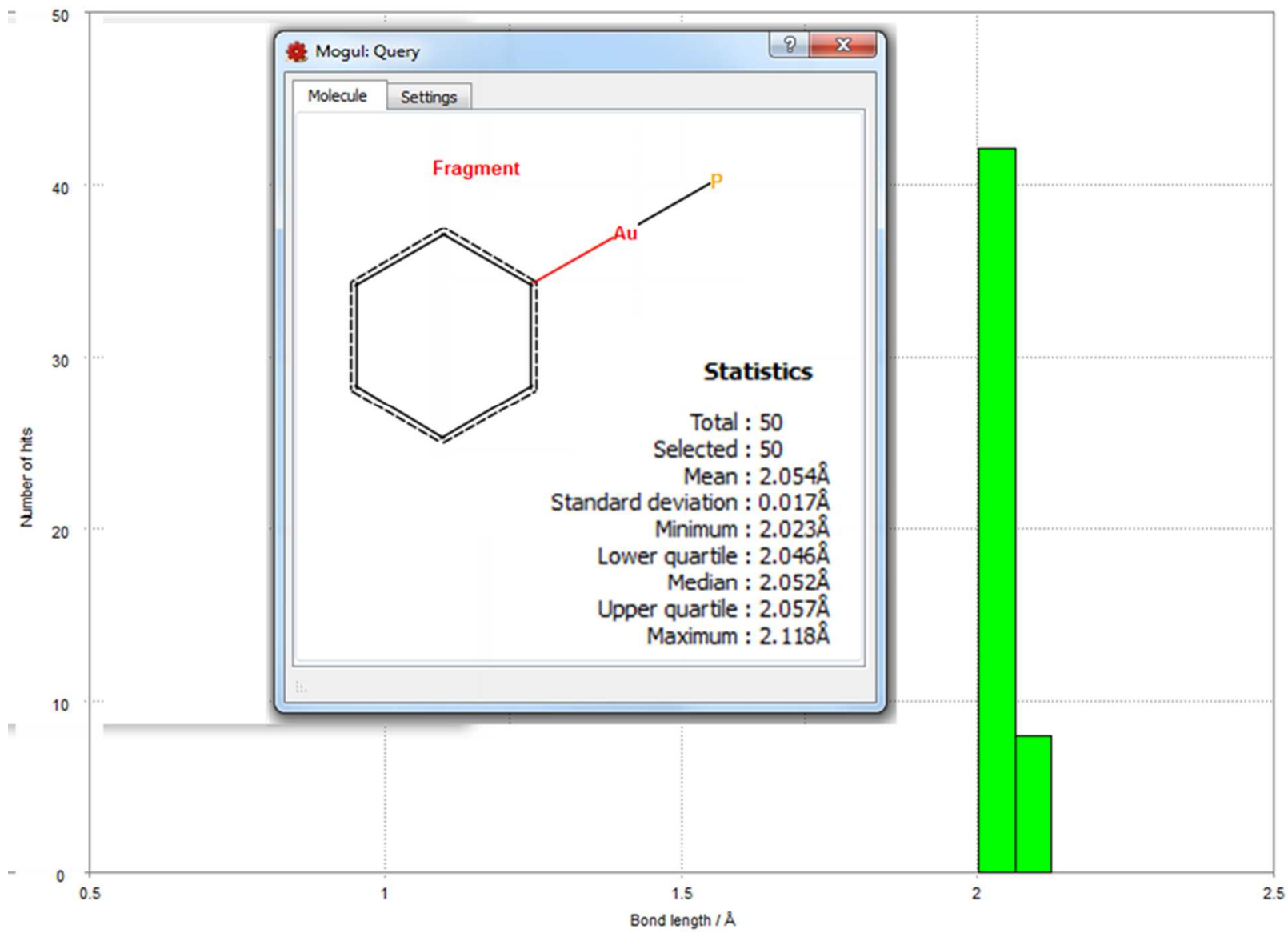

Figure S23. Histogram of the $\mathrm{Au}-\mathrm{C}_{\mathrm{Ph}}$ bond distances found in the Cambridge Structural Database using Mogul 1.7.1. The fragment used in the search and the statistics are shown in the inset. 